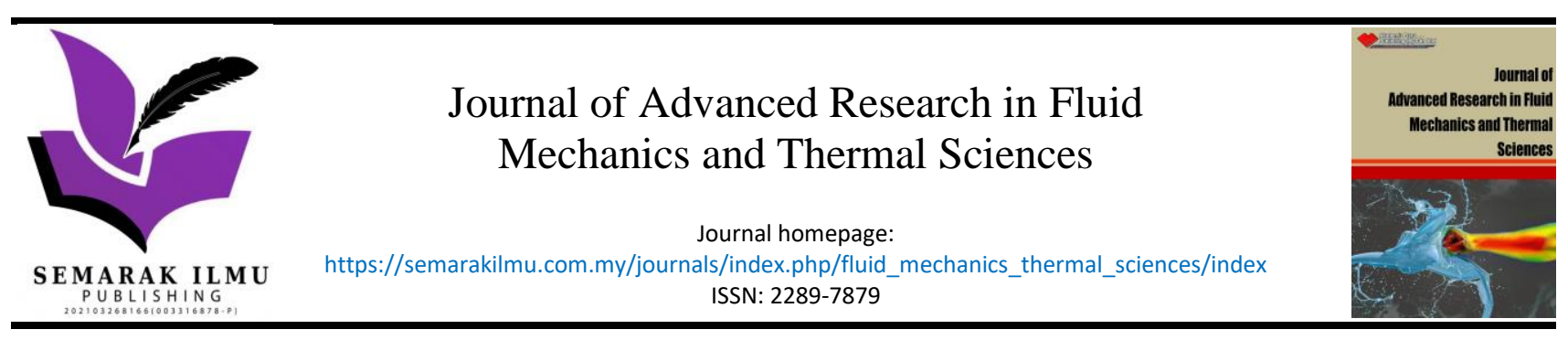

\title{
Numerical Analysis on the Crosswind Influence Around a Generic Train Moving on Different Bridge Configurations
}

\author{
Izuan Amin Ishak ${ }^{1,2^{*}}$, Nurshafinaz Maruai ${ }^{3}$, Fadhilah Mohd Sakri' ${ }^{4}$, Rahmah Mahmudin ${ }^{1}$, Nor \\ Afzanizam Samiran ${ }^{1}$, Syabillah Sulaiman ${ }^{1}$, Shaiful Fadzil Zainal Abidin ${ }^{1}$, Nik Normunira Mat Hassan ${ }^{1}$ \\ 1 Faculty of Engineering Technology, Department of Mechanical Engineering Technology, Universiti Tun Hussein Onn Malaysia, EDU Hab Pagoh, \\ 84600 Johor, Malaysia \\ Sustainable Engineering Technology Research Centre, Universiti Tun Hussein Onn Malaysia, EDU Hab Pagoh, 84600 Johor, Malaysia \\ 3 Malaysia Japan International Institute of Technology, Universiti Teknologi Malaysia, 54100 Kuala Lumpur, Malaysia \\ 4 Universiti Kuala Lumpur - Malaysia Institute of Aviation Technology, Jalan Jenderam Hulu, Kampung Jenderam Hulu, 43900 Sepang, Selangor, \\ Malaysia
}

\section{$\begin{array}{ll}\text { ARTICLE INFO ABSTRACT } & \text { ABST }\end{array}$}

\section{Article history:}

Received 26 July 2021

Received in revised form 27 October 2021

Accepted 29 October 2021

Available online 30 November 2021

\section{Keywords:}

Aerodynamic loads; bridge; crosswind; flow regime; flow structure; generic train

\begin{abstract}
In this article, a numerical approach is applied to study the flow regimes surround a generic train model travelling on different bridge configurations under the influence of crosswind. The bridge is varies based on the different geometry of the bridge girder. The crosswind flow angle $(\Psi)$ is varied from $0^{\circ}$ to $90^{\circ}$. The incompressible flow around the train was resolved by utilizing the Reynolds-averaged Navier-Stokes (RANS) equations combined with the SST k- $\omega$ turbulence model. The Reynolds number used, based on the height of the train and the freestream velocity, is $3.7 \times 10^{5}$. In the results, it was found that variations of the crosswind flow angles produced different flow regimes. Two unique flow regimes appear, representing (i) slender body flow behaviour at a smaller range of $\Psi$ (i.e. $\psi \leq 45^{\circ}$ ) and (ii) bluff body flow behaviour at a higher range of $\psi$ (i.e. $\psi \geq 60^{\circ}$ ). As the geometries of the bridge girder were varied, the bridge with the wedge girder showed the worst aerodynamic properties with both important aerodynamic loads (i.e. side force and rolling moment), followed by the triangular girder and the rectangular girder. This was due to the flow separation on the windward side and flow structure formation on the leeward side, both of which are majorly influenced by the flow that moved from the top and below of the bridge structures.
\end{abstract}

\section{Introduction}

The lateral stability of a train is an important safety issue as it is largely dependent on aerodynamic load caused by crosswinds. The aerodynamic load can account for up to $63 \%$ of the unloading of the wheels [1]. Side force, lift force, and rolling moment are the significant aerodynamic loads to consider while examining the train's lateral stability (overturning or rollover) in crosswinds. Because trains are increasingly being built with lightweight materials to attain higher speeds and are turning toward more comfortable carriages to accommodate a large number of people, these trends

\footnotetext{
* Corresponding author.

E-mail address: izuan@uthm.edu.my
}

https://doi.org/10.37934/arfmts.89.2.7698 
suggest that any train must have considerably superior handling and stability. However, using lightweight materials to build a train that can reach faster speeds comes at the expense of the vehicle's crosswind stability [2].

Alertness on safety factors especially the rail vehicle crosswind stability has grown significantly in the community [2-10]. The fact that there have been few significant cases in recent years where strong winds are assumed to be the primary cause of derailment emphasises the need for more investigation [11]. There are tremendous amounts of wind-related railway vehicle incidents that have been documented in the past (e.g., Japan, Belgium, Italy, Switzerland, and China) [12-14]. The majority of them occurred on narrow gauge rails with much greater wind speeds [13]. These are the reasons why it is critical to investigate the crosswind stability of railway vehicles, which are unavoidably affected by wind conditions.

According to previous research, the train's aerodynamic properties under crosswind are heavily influenced by the infrastructures over which it travels, such as embankments, bridges and viaducts [15-20]. There are several situations where the railings must be raised above the ground due to an unavoidable topographical surface. Additional infrastructure can be used to provide a platform for the operation of rail vehicles. The aerodynamic stability of the vehicle will be harmed when the railway level is increased to a greater altitude. High bridges add significantly to the cumulative risk of mechanical safety violations [21]. The wind speed increases with the height from the ground to a higher elevation, and consequently affects the force exerted by the train. Trains travelling on bridges have considerably increased aerodynamic forces, which may increase the chance of trains derailing and overturning $[3,15,22,23]$.

There was not much research on the impact of infrastructure on train aerodynamics up until now. The known findings are largely based on the experimental study [15,24,25]. Suzuki et al., [15] conducted an experiment to look at the consequences of varying bridge girder thicknesses. Their findings reveal that increasing the thickness of the bridge girder increases the aerodynamic side force coefficient. Despite this, Cheli et al., [24] and Bocciolone et al., [25] conducted experimental investigations to examine the Technical Specification for Interoperability (TSI) infrastructure scenario based on train crosswind activities. As an outcome, Bocciolone et al., [25] discovered that for small yaw angles, the embankment configuration's side force and lift force coefficients are somewhat greater than those recorded on bridges. This is due to the varying wind speeds at the infrastructure's top. The embankment configuration generates flow acceleration and overpressure on the train at low crosswind yaw angles, but the bridge configuration appears to be more essential in terms of both the side and lift force coefficients for greater yaw angles [25].

Only a few numerical studies have been conducted to investigate the aerodynamics of trains travelling over infrastructure. As a result, the amount of data accessible for reference in this study is quite limited. Diedrichs et al., [3] in their study focused exclusively on the effect of an embankment with a fixed height and slope. According to their research, the 6-meter high embankment reduced the allowed crosswind speed by about $20 \%$ when compared to the level ground condition. The maximum side force for a rail vehicle travelling on embankment increases at $\psi=50^{\circ}$, according to Zhou et al., [26], and the overturning moment coefficient follows a similar pattern. Xiang et al., [27], on the other hand, investigated the critical speed of a HST operating on a bridge in strong crosswinds. Their research compared a train travelling in a straight path across a bridge against a train moving on a curved track. Xiang et al., [28] investigated the impacts of installing wind barriers on the bridge and how they affected the train's aerodynamic performance. As a result, the wind barriers reduce the vehicle's aerodynamic loads, notably by lowering positive pressure on the train's body's windward side and negative pressure on the roof side [27]. 
In the context of the train's geometry, based on past investigation, some have considered much more complex geometries by considering additional structures such as a front spoiler, bogies, and pantograph, while others define their structures (e.g. the shape of the train) in a more simplified way. Outcomes maintain that the complex geometries of train model did not influence much on the end results of the simulations [29-31].

To summarise, aerodynamic factors are regarded as one of the most important criteria that must be thoroughly addressed. However, focusing just on the vehicle's design is insufficient, especially when there are additional external issues to consider, such as crosswind and varied platform conditions, both of which make the study of aerodynamic phenomena more fascinating. It is anticipated that by addressing these related aerodynamic concerns, the train's operational safety would be ensured. The goal of this research is to examine aerodynamic features such as aerodynamic load and physic flow structure, as well as how the results relate to the occurrence of two flow regimes (i.e., slender body flow regime and bluff body flow regime) on a generic train model.

This paper has been organized in the following way. Section 2 discusses the computational setup inclusive of the detailed model (train and its infrastructures), domain description, boundary condition and solution methodology. Section 3 prevails the grid convergence study which is done by a systematic method using the Grid Convergence Index $(\mathrm{GCl})$ and the Richardson Extrapolation. Next, Section 4 discusses the outcome of the results from the present study. This include (a) the aerodynamic loads (b) the physic of flow structure and (c) the pressure coefficient in which all cases are deliberated comprehensively. Finally, the conclusion is stated in the last section of the article.

\section{Methodology}

\subsection{Pressure Distribution}

The magnitude of a train's aerodynamic loads is affected by the direction of the effective crosswind. In this case, the effective crosswind is defined as the vector summation between the train speed $\left(U_{t}\right)$ and the wind velocity $\left(U_{w}\right)$ as shown in Figure 1.

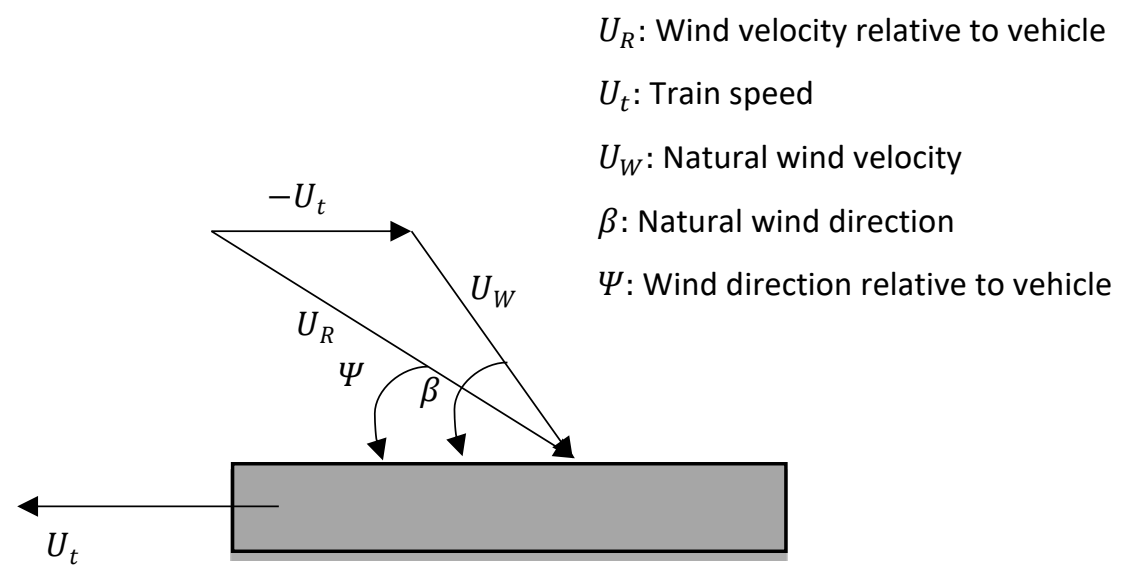

Fig. 1. Components of natural wind velocity relative to the vehicle

\subsection{Train Model Description}

The train model utilised in this study is a generic train model with a blunt nose, as seen in Figure 2. This particular model geometry was chosen to conduct validation and comparison research with prior investigators (experimentally by Sakuma et al., [32] and numerically by Osth et al., [29]). As 
shown in Figure 2(a)-(b), the leading side and top edges on the front are rounded using an elliptical shape with a main axis in the ellipse length of $0.07 \mathrm{H}$ and a minor axis length of $0.04 \mathrm{H}$. Figure 2 (c) on the other hand shows the side and top edges of the vehicle's rear end that are rounded with a circular radius of $0.107 \mathrm{H}$. Both front and rear bottom edges are not rounded and thus sharp. The length of the train is $7 H$ while the width and height are both equal to $H$. ( $W=H=0.56 \mathrm{~m})$.

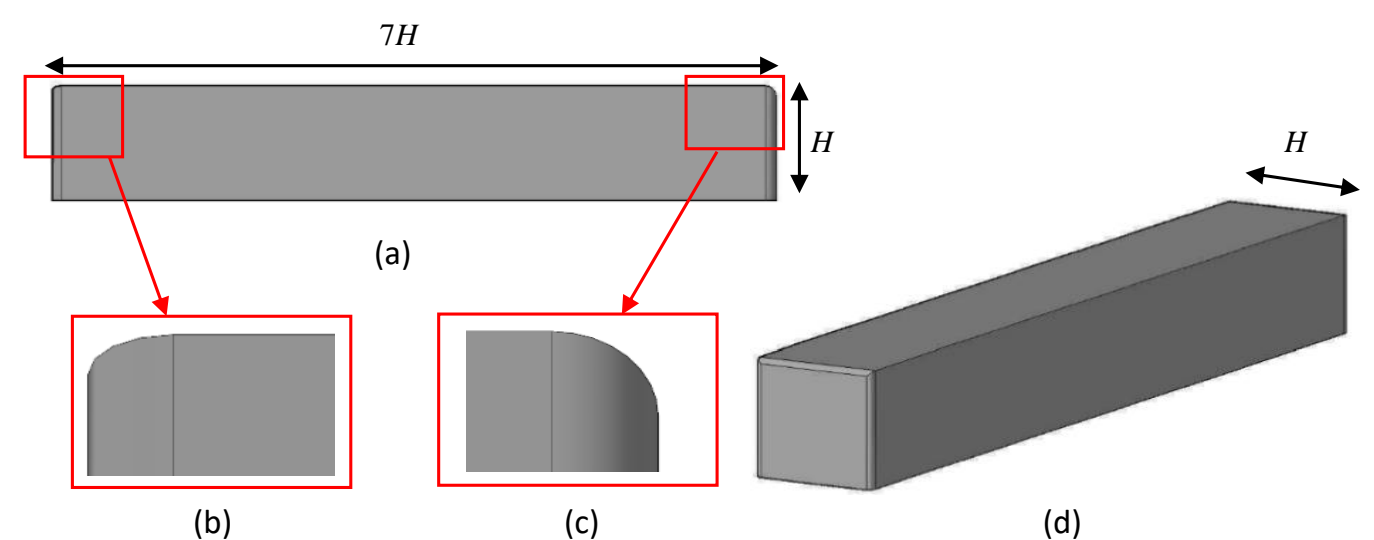

Fig. 2. The geometry of the train model with blunt nose shape. (a) Side view (b) Close view of the front corner with an elliptic rounding (c) Close view of the rear corner with a circular rounding $(d)$ Isometric view

\subsection{Bridge Description}

The test cases can be divided into two categories of surfaces on which the generic train model is travelling: flat ground (FGC) and bridge. Figure 3 shows the vehicle on top of a bridge. There are three shapes of the bridge girder: (a) rectangular girder shape, (b) triangular girder shape, and (c) wedge girder shape. All models have the same width to thickness ratio $(B / D)$ which is equal to $5: 1$ and the height $(h)$ is fixed at 6 meters. This parameter is expected to give different aerodynamic characteristics of the train $[15,33,34]$. The first (a) and second (b) girder shapes represent a more basic construction shape whilst the third (c) shape (i.e. wedge girder) is a more realistic shape resembling the so-called the 'Great Belt East Bridge' deck shape that connects Sprog $\varnothing$ and Zealand in Denmark. These simplified geometries of the bridge girders are chosen to give an overview of flow behaviour as the crosswind passes through it.

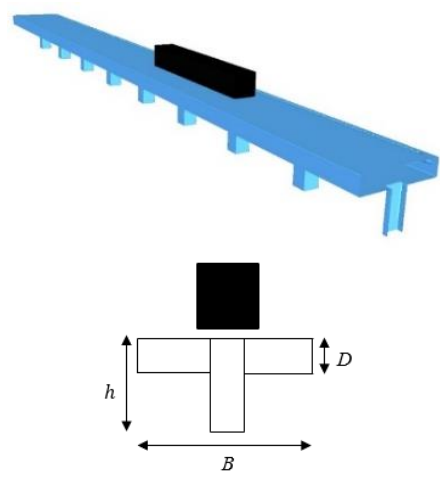

(a)
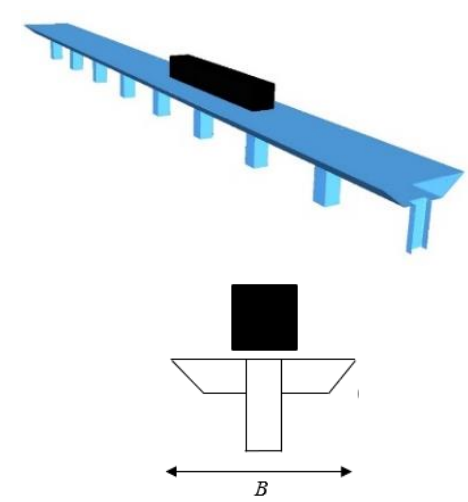

(b)

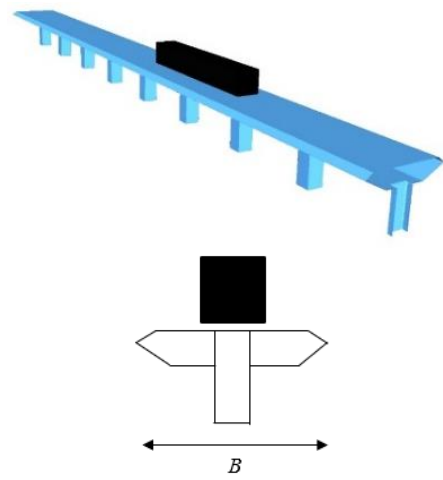

(c)

Fig. 3. Shapes of the bridges (a) rectangular girder (b) triangular girder (c) wedge girder. The inset pictures represent the cross-section views 


\subsection{Computational Domain}

General guidelines on the distances between the inlet and the vehicle as well as the vehicle with the outlet follow the proposed length parameter based on a previous investigation made. The computational domain is based on the reference $[25,35,36]$ which specify the appropriate distances from the inlet to train model is equal to $8 \mathrm{H}$ and the distance from train model to the outlet is equal to $21 \mathrm{H}$. Figure 4 shows the placement of the train model travelling on the flat ground case. A similar setup was applied for the bridge cases.

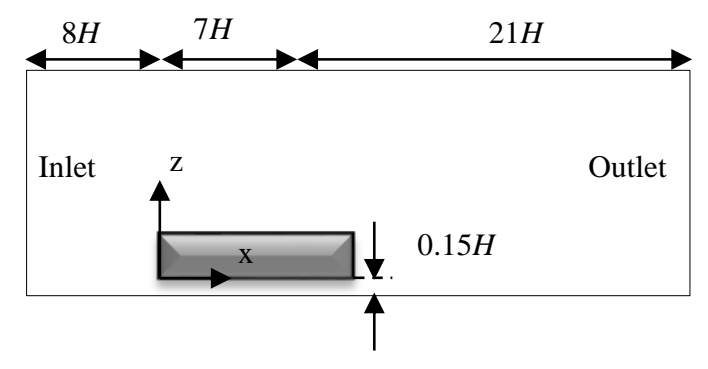

(a)

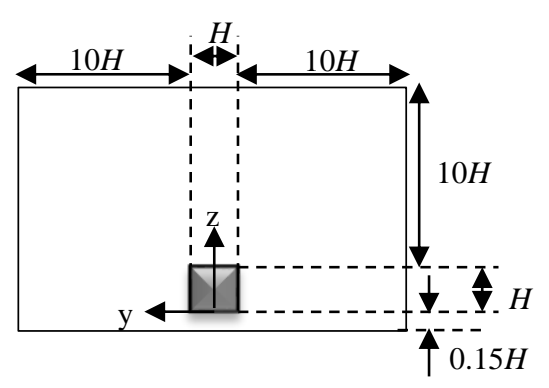

(b)

(a) $l_{1}$ $7 H$

(b) $l_{2}$

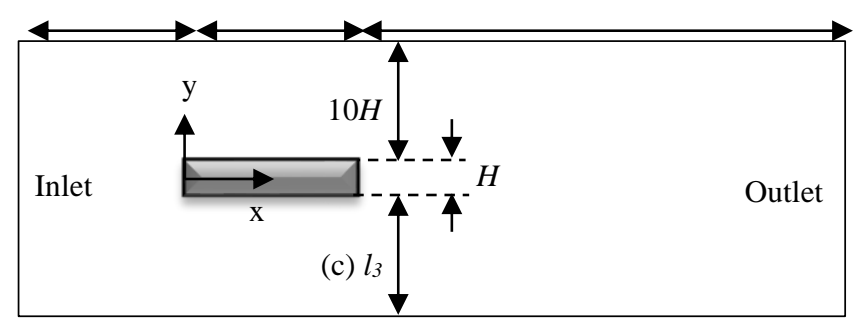

(c)

Fig. 4. The computational domain used in the numerical investigation for $0^{\circ}$ yaw angle condition. (a) Side view (b) Front view (c) Top view (Sketch only and not following the actual scale)

To simulate the usual real train situation, the generic train models are positioned $0.15 \mathrm{H}$ above the ground $[17,37]$. The model of the train is also placed far enough away from the top and sidewalls $(10 \mathrm{H})$ to reduce near-wall effects. Figure 4 depicts the domain for the case of $0^{\circ}$ yaw angle. For the rest of the crosswind conditions $\left(15^{\circ}, 30^{\circ}, 45^{\circ}, 60^{\circ}, 75^{\circ}\right.$ and $\left.90^{\circ}\right)$, computational domains will follow arrangements as presented in Table 1 based on the view from the top. This is because the effective crosswind progressively changes in the lateral direction in relation to the train location (the crosswind yaw angle ranges from $0^{\circ}$ to $90^{\circ}$ ). As a result of these modifications, the nearest inlet is always equal to or greater than $8 \mathrm{H}$, and the nearest outlet is always equal to or more than $21 \mathrm{H}$.

Table 1

Domain size parameter based on Figure 4(c) for different cases of crosswind yaw angles

\begin{tabular}{llll}
\hline Yaw Angle & $I_{1}$ & $I_{2}$ & $I_{3}$ \\
\hline $0^{\circ}$ & $8 H$ & $21 H$ & $10 H$ \\
$15^{\circ}$ & $8.4 H$ & $20.3 H$ & $10.5 H$ \\
$30^{\circ}$ & $8.8 H$ & $18.2 H$ & $12.8 H$ \\
$45^{\circ}$ & $9.2 H$ & $14.9 H$ & $16.1 H$ \\
$60^{\circ}$ & $9.6 H$ & $10.5 H$ & $20.5 H$ \\
$75^{\circ}$ & $10 H$ & $10 H$ & $21 H$ \\
$90^{\circ}$ & $10 H$ & $10 H$ & $21 H$ \\
\hline
\end{tabular}




\subsection{Boundary Condition}

Figure 5 depicts visualisations of the boundary conditions. The following are the details of the boundary conditions:

i. Inlet: Uniform velocity, which represents the free stream velocity $\left(U_{\infty}\right)$ is applied in the $x$ direction. For crosswind conditions $\left(\psi>0^{\circ}\right)$, the resultant wind velocity will follow the component velocity vector based on the effective crosswind.

ii. Ground plane: The boundary type of moving wall is applied with the velocity component in the $x$-direction equal to the inlet velocity $\left(U_{\infty}\right)$ to prevent the development of the boundary layer on the ground plane.

iii. Outlet: The homogenous Neumann boundary condition is applied at the outlet, meaning that the pressure gradient is equal to zero.

iv. Lateral side and roof plane: The patch type boundary condition with a freestream value similar to the inlet is used. For crosswind conditions $\left(\Psi>0^{\circ}\right)$, the right plane will become an inlet whilst the left plane will be transformed into an outlet boundary condition.

v. Train model surface: The no-slip condition is used.

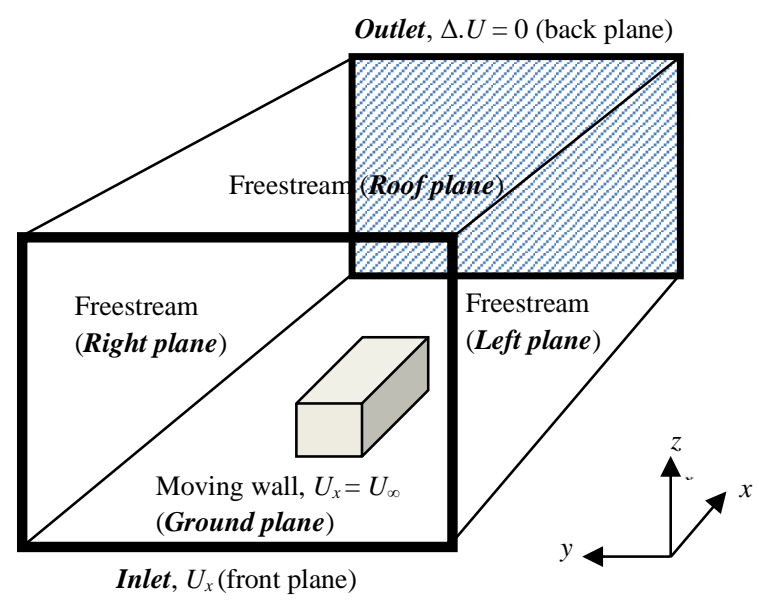

Fig. 5. Boundary conditions used in the numerical investigation (not following the actual scale)

\subsection{Solution Methodology}

The flow around the train has been considered incompressible and is obtained by solving the incompressible form of the Reynolds Averaged Navier-Stokes (RANS) equations. The two equations which are Continuity and Navier-Stokes equations for the incompressible flow as follows:

$\frac{\partial U_{i}}{\partial x_{i}}=0$

$\frac{\partial U_{i}}{\partial t}+U_{j} \frac{\partial U_{i}}{\partial x_{j}}=-\frac{1}{\rho} \frac{\partial P_{i}}{\partial x_{i}}+\frac{\partial}{\partial x_{j}}\left(\mu \frac{\partial U_{i}}{\partial x_{j}}-\rho \overline{u_{\imath}^{\prime} u_{\jmath}^{\prime}}\right)$ 
in which indices $i, j=1,2,3$ refer to the streamwise $-x$, cross-stream -y and -z-direction in a Cartesiancoordinate system respectively. $U_{i}$ and $P_{i}$ are the time-averaged terms, while $u_{i}^{\prime}$ is the fluctuation terms of velocity and $p_{i}^{\prime}$ is the fluctuation terms of pressure.

The OpenFOAM CFD software programme is utilised in this work to solve the governing equations. Table 2 shows the detailed numerical parameters used throughout the whole case study.

\section{Table 2}

Numerical methods used in OpenFOAM

\begin{tabular}{|c|c|c|c|}
\hline \multicolumn{2}{|c|}{ Discretization } & Scheme & Description \\
\hline \multicolumn{2}{|c|}{ Time } & steadyState & - \\
\hline \multirow[t]{3}{*}{ Spatial } & Gradient & Central differencing & 2nd order central differencing \\
\hline & Divergence & QUICKV & 3rd order \\
\hline & Laplacian & $\begin{array}{l}\text { Gauss linear differencing } \\
\text { scheme }\end{array}$ & $2^{\text {nd }}$ order unbounded \\
\hline \multicolumn{2}{|c|}{ Pressure-velocity coupling } & SIMPLE & Used as a steady flow algorithm \\
\hline \multicolumn{2}{|c|}{ Turbulence models } & RANS $k-\omega$ & Shear-Stress-Transport (SST) \\
\hline \multirow{3}{*}{$\begin{array}{l}\text { Wall } \\
\text { functions }\end{array}$} & $k$ & kqRWallFunction & Acts as a zero-gradient condition for modelled $k$ \\
\hline & $\omega$ & omegaWallFunction & Automatic wall functions condition for $\omega$ \\
\hline & $v_{t}$ & nutkWallFunction & $\begin{array}{l}\text { Generates a near-wall profile for } v_{t} \text { based on } \\
\text { modelled } k\end{array}$ \\
\hline
\end{tabular}

\section{Validation Study}

\subsection{Grid Convergence Study}

To examine the influence of grid dependency, solutions to three distinct grid refinements representing fine, medium, and coarse grids are simulated. As indicated in Table 3 , the various grid resolutions are carefully determined depending on the grid refinement ratio $(r)$. Shown in Figure 6 is the detail mesh for the fine grid resolution. The train model shown below was given pillar support for a comparative study with earlier research on the same train model by Sakuma et al., [32] and Osth et al., [29].

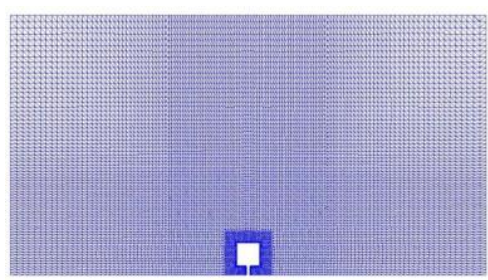

(a)

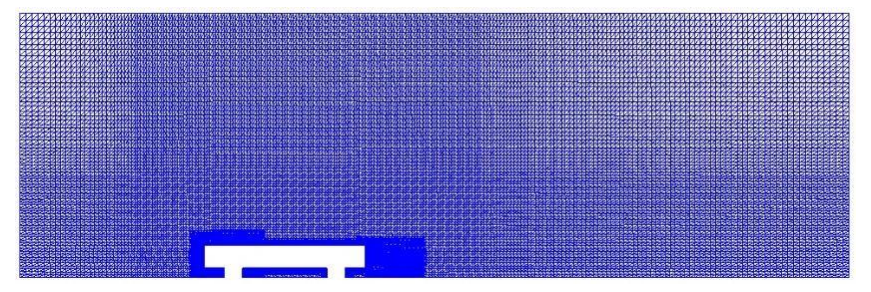

(b)

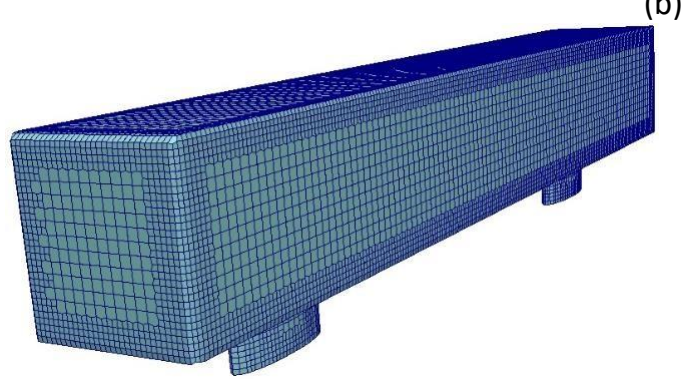

(c)

Fig. 6. Detail of mesh for the fine grid resolution. (a) front view at $1 \mathrm{H}$ from front nose cross-section (b) side view at the middle plane cross-section, and (c) on the train mode 
According to Celik et al., [37], the ratio must be larger than 1.3. Due to the non-uniformity of the meshes, the grid refinement ratio is determined using the average grid size $\left(h_{\text {ave }}\right)$. The grid refinement ratio $(r)$ and the average cell size $\left(h_{\text {ave }}\right)$ can be calculated as follows

$r_{21}=\frac{h_{2}}{h_{1}}$

$r_{32}=\frac{h_{3}}{h_{2}}$

$h_{\text {ave }}=\left[\frac{1}{N} \sum_{i=1}^{N}\left(\Delta V_{i}\right)\right]^{\frac{1}{3}}$

where $\Delta V_{i}$ is the volume of the $i^{\text {th }}$ cell and $N$ is the total number of cells used for the computations.

Table 3

Grid parameter for the case I, II and III where subscripts 1, 2 and 3 represents case I, II and III respectively

\begin{tabular}{llll}
\hline CASE & I (Fine) & II (Medium) & III (Coarse) \\
\hline Total No. of Cells, $\boldsymbol{N}$ & $2,114,715$ & 951,838 & 359,838 \\
Average cell size, $\boldsymbol{h}_{\text {ave }}$ & 0.0895 & 0.1168 & 0.1615 \\
Average, $\boldsymbol{y}^{+}$ & 81.76 & 83.28 & 113.59 \\
Refinement ratio, $\boldsymbol{r}$ & $r_{21}=1.31$ & & $r_{32}=1.38$ \\
\hline
\end{tabular}

The Richardson Extrapolation $\left(f_{R E}\right)$ and Grid Convergence Index are used to evaluate the Grid Convergence Index $(\mathrm{GCl})$. The value anticipated from the Richardson Extrapolation $\left(f_{R E}\right)$ is, by definition, the value that would arise if the cell grid size tended to zero $(h \rightarrow 0)$. Alternatively, the $\mathrm{GCl}$ value indicates that the percentage of the computed value is away from the value of the asymptotic numerical value. It shows an error band on how far the solution is from the asymptotic value and how much the solution would change with further refinement of the grid This is achieved by comparing $\mathrm{GCl}$ findings for various parameters at different mesh resolution levels. Both of these indicators are essential in accessing a grid convergence study [38-40].

According to the results, monotonic convergence criteria $(R)$ are achieved since $0<R<1$ (refer to Table 4). Figure 7 clearly shows how the value of the selected parameter steadily progressed in a converging pattern towards the Richardson extrapolated value. This also explained that the error, due to grid convergence, gradually decreased and the fine grid resolution $\left(\mathrm{GCl}_{21}\right)$ had a value of less than $1 \%$ for almost all the compared parameters. To summarise, because the $\mathrm{GCl}$ reduction from the coarser grid $\left(\mathrm{GCl}_{32}\right)$ to the finer grid $\left(\mathrm{GCl}_{21}\right)$ is very large, the grid-independent solution is nearly achieved, implying that additional grid refinement will not have a significant influence on the outcomes of flow simulation $[7,41]$.

\section{Table 4}

Grid Convergence Index (GCI) for different parameters

\begin{tabular}{llll}
\hline CASE & $R$ & $\mathrm{GCl}_{32}(\%)$ & $\mathrm{GCl}_{21}(\%)$ \\
\hline$C_{d}$ mean & 0.133 & 0.5542 & 0.1122 \\
Stagnation Pressure & 0.2932 & 0.3262 & 0.1307 \\
Base Pressure & 0.255 & 2.9056 & 1.0496 \\
Wake Length & 0.170 & 1.1946 & 0.2969 \\
\hline
\end{tabular}




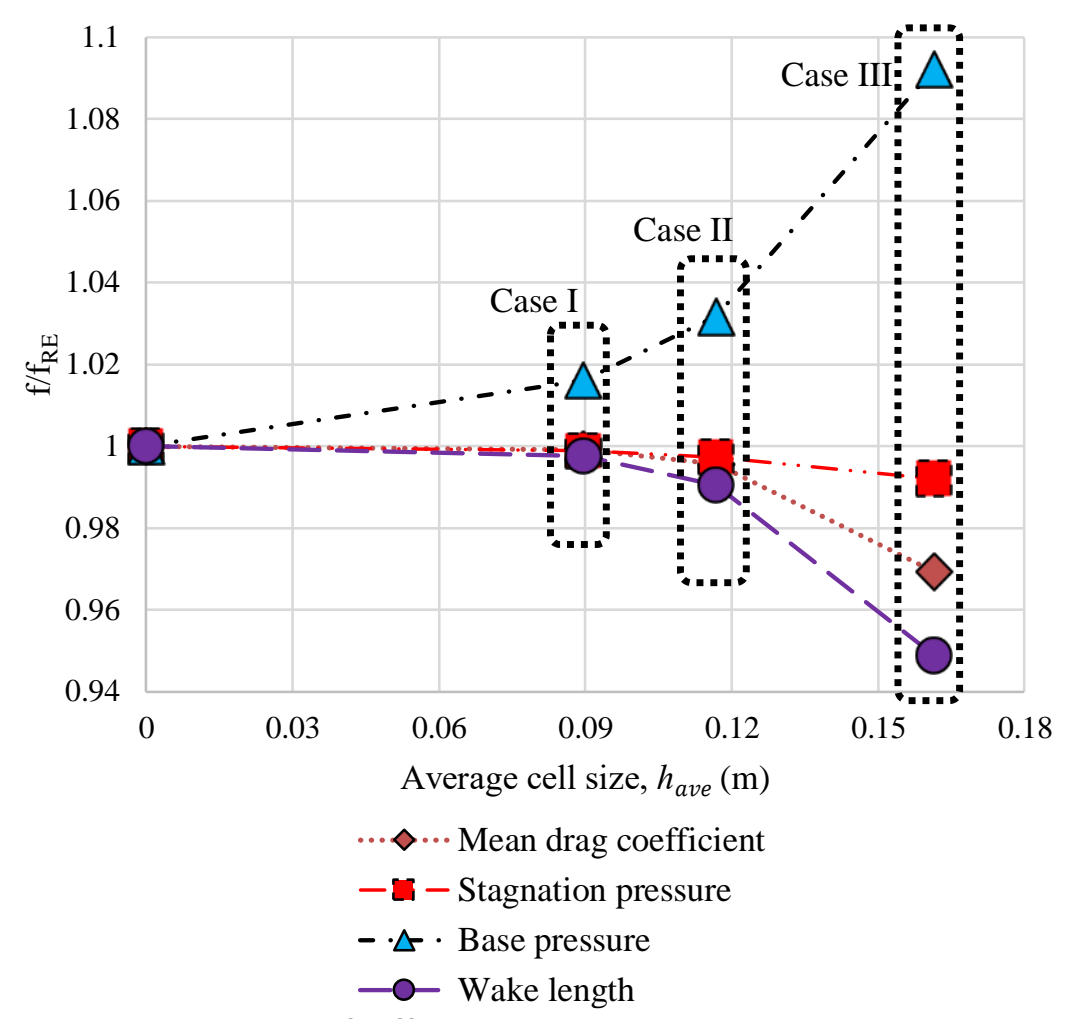

Fig. 7. Comparison of different integral parameters normalized by the extrapolated, between three grid solutions and Richardson extrapolation estimation

\section{Results and Discussions}

\subsection{Aerodynamic Loads}

In this section, analysis of quantitative data based on aerodynamic loads properties such as the coefficient of side force $\left(C_{s}\right)$, drag force $\left(C_{d}\right)$, lift force $\left(C_{l}\right)$, and rolling moment $\left(C_{R L}\right)$ are compared between each case as shown in Figure 8-11.

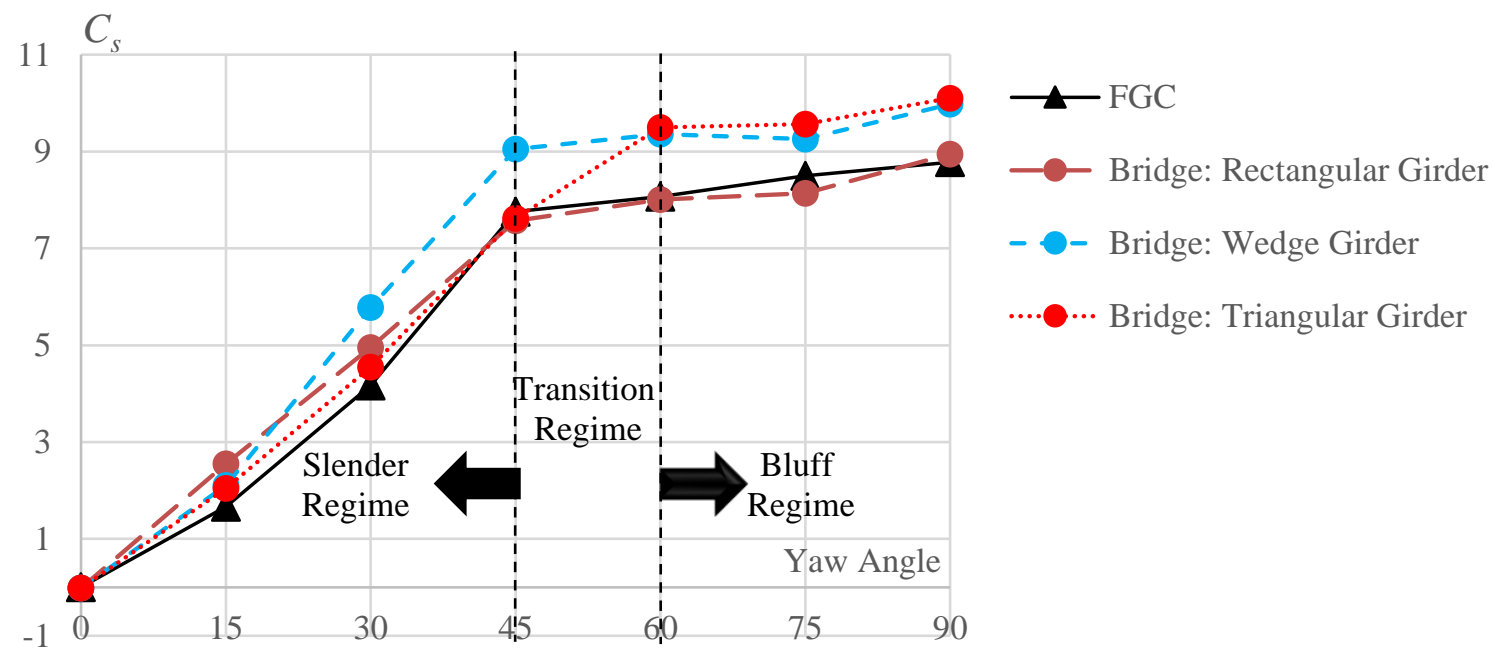

Fig. 8. Comparison of the side force coefficient $\left(C_{s}\right)$ with respect to different crosswind conditions for the train moving on FGC and various bridge platforms 


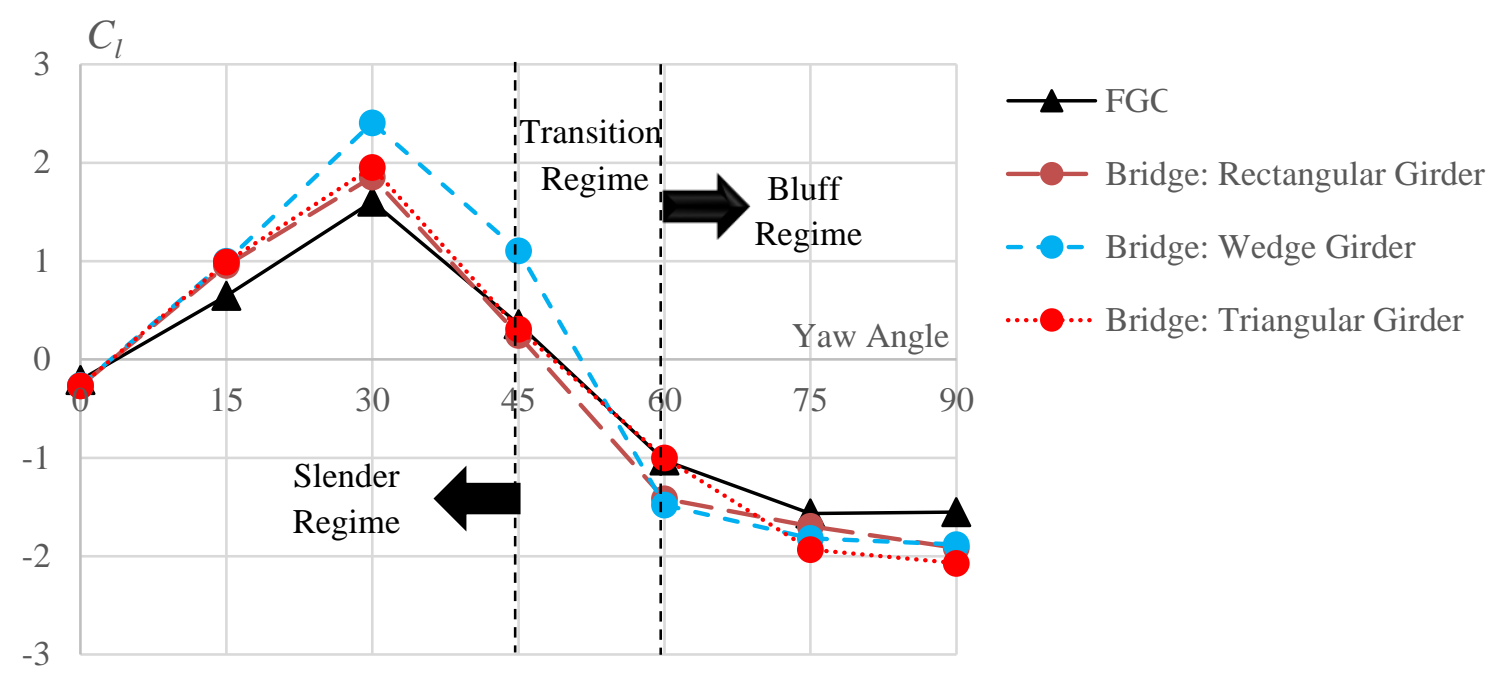

Fig. 9. Comparison of the lift force coefficient $\left(C_{1}\right)$ with respect to different crosswind conditions for the train moving on FGC and various bridge platforms

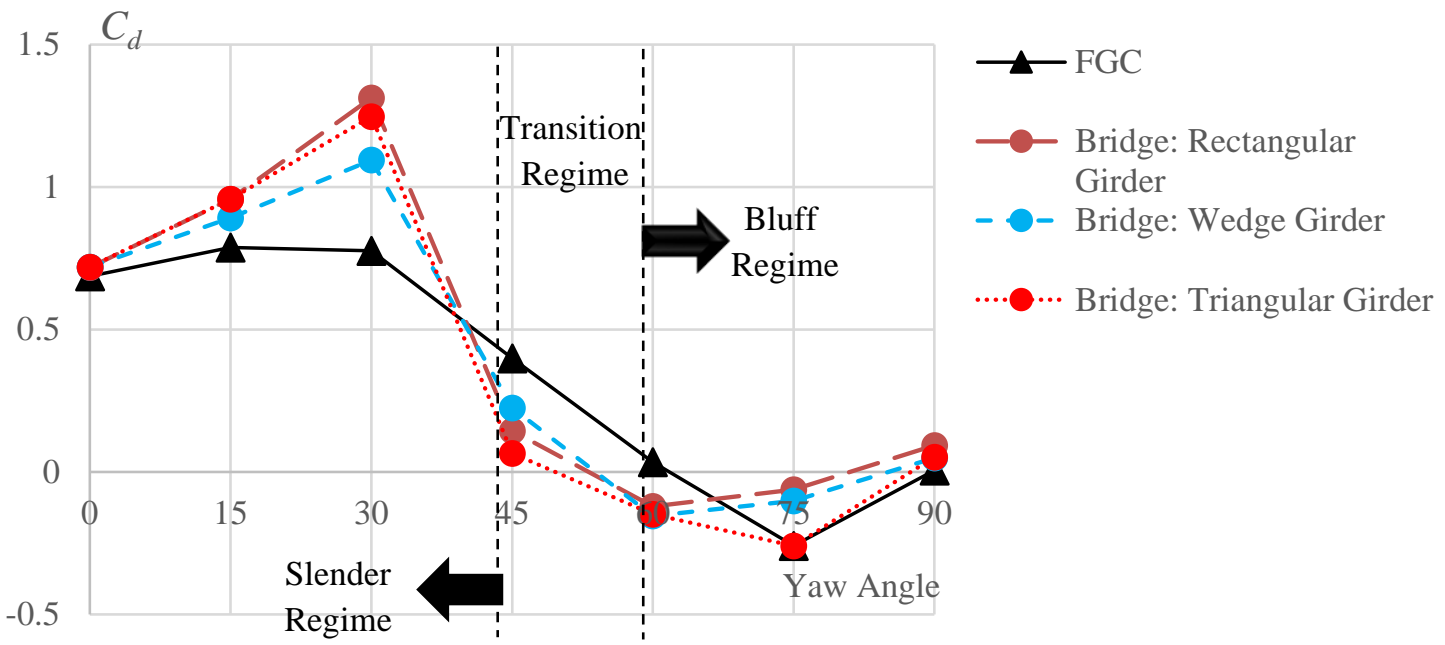

Fig. 10. Comparison of the drag force coefficient $\left(C_{d}\right)$ with respect to different crosswind conditions for the train moving on $\mathrm{FGC}$ and various bridge platforms

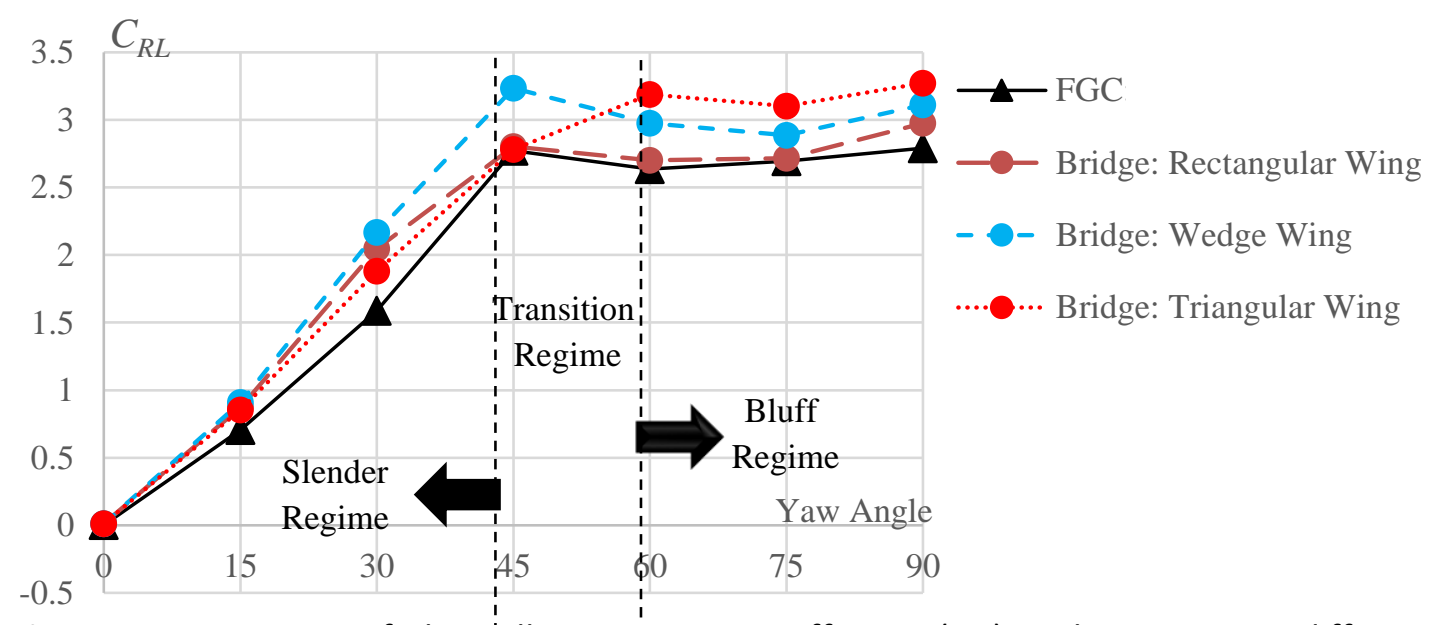

Fig. 11. Comparison of the rolling moment coefficient $\left(C_{R L}\right)$ with respect to different crosswind conditions for the train moving on FGC and various bridge platforms 


\subsubsection{Side force}

Figure 8 shows the effects of wind directions on the side force coefficient, $C_{s}$. The change in the $C_{s}$ value can be grouped into different characteristics of flow regimes. The first regime describes the slender body flow behaviour where the $C_{s}$ changes almost linearly with the yaw angle i.e. at the low range of yaw angles (i.e. $\psi \leq 45^{\circ}$ ). In the higher range of yaw angles, the second regime (describing the bluff body flow behaviour) is observed where $C_{s}$ is almost not affected by the change in the crosswind direction. It is also important to note that at the transition regime (i.e. $45^{\circ} \leq \Psi \leq 60^{\circ}$ ), the flow is in the conversion phase between the slender to bluff body flow behaviour. Among the different bridge girder cases, the one with a wedge girder shape shows the lowest $C_{s}$ value followed by the triangular girder and the rectangular girder. At $\psi=30^{\circ}$, the $C_{s}$ for the bridge with the wedge girder starts to differ from others (about 38.7\% higher than the FGC) and the difference in these values is almost consistent throughout the rest of yaw angle conditions. Interestingly, for the triangular girder, the $C_{s}$ for slender body flow regime extends up to $\psi=60^{\circ}$ which is higher than all the other cases that display the slender body flow regime of only up until $\psi=45^{\circ}$. This can be observed from the graph that shows an almost linear increment until $\psi=60^{\circ}$ before the $C_{s}$ starts to stabilize. It is also important to notice that the $C_{s}$ of the rectangular girder especially in the bluff body flow regime is almost similar to that of the FGC. This emphasizes that the rectangular girder shape is the best bridge design structure for the deck shape, especially when considering the lateral aerodynamic loads $\left(C_{s}\right)$.

\subsubsection{Lift force}

As shown in Figure 9, the $C_{\text {/ }}$ patterns with respect to the crosswind for the train moving on bridges are also relatively similar when the train is moving on a FGC. However, at $\psi=30^{\circ}$, the maximum value is much higher compared to the baseline case. The peak value of $C_{l}$ for the case of the bridge with wedge girder shape magnifies about $50 \%$ from the FGC at $\psi=30^{\circ}$. At $\psi=45^{\circ}$, the value of $C_{l}$ drops. At larger yaw angle conditions $\left(\psi \geq 60^{\circ}\right)$, in the bluff body flow regime, it is observed that all cases exhibit similar results of much lower $C_{l}$ values. For the different bridge girder design comparisons, the wedge girder once again demonstrates the highest $C_{l}$ value is followed by the triangular girder and the rectangular girder (for the lowest $C_{1}$ ). In general, simple generalization can be perceived in the trend of $C_{/}$with yaw angle variations. In the slender body flow regime, the graph shows a fluctuating increase to a maximum value before the $C_{\text {I }}$ decreases, whilst in the bluff body

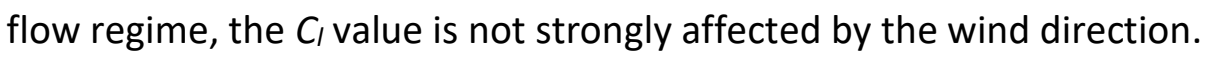

\subsubsection{Drag force}

It is obvious to see that the flow regimes can also be observed from the changes of the $C_{d}$ with the yaw angle as shown in Figure 10. Generally, the graph pattern is almost similar to the $C_{l}$ as shown in Figure 9. There is an increasing and decreasing trend in the slender body flow regime, and the value is less influenced by the crosswind conditions in the bluff body flow regime. Hence, it is deduced that the effect of drag is a major influence in the slender body flow regime. This trend agrees well with Suzuki et al., [15] and Baker [42] which mentioned that the largest drag forces associated with the skin friction drag exist in the slender body flow regime. From Figure 10, an increasing trend of the $C_{d}$ occurs from $\psi=0^{\circ}$ until $\psi=30^{\circ}$ before the value starts to drop. Among the cases in the bridge girder scenario, the rectangular girder achieves the worst drag issue followed by the triangular girder 
and the wedge girder. By comparison with the FGC, the maximum value recorded at $\psi=30^{\circ}$ increases about $20 \%$ to $50 \%$ higher for the bridge cases.

\subsubsection{Rolling moment}

The shape of the graph for the rolling moment resembles the side force coefficient $\left(C_{s}\right)$ graph as the value of $C_{R L}$ increases until it reaches an optimal value at $\psi=45^{\circ}$. In Figure 11, the $C_{R L}$ value shows a nearly linear increase at a low range of yaw angles (i.e. $\psi \leq 45^{\circ}$ ) and virtually a steady value at high yaw angles (i.e. $\psi \geq 60^{\circ}$ ). In the transition flow regime, it is notable that after it reaches the maximum point at $\psi \leq 45^{\circ}$, the $C_{R L}$ value slightly drops before it becomes stable. Since the triangular girder has an extended slender body flow region (as shown in Figure 11), the peak value of $C_{R L}$ is recorded at $\psi$ $=60^{\circ}$ whilst others are at $\Psi=45^{\circ}$. In the slender body flow regime, the wedge girder case recorded the worst $C_{R L}$ condition. Conversely, in the bluff body flow regime, the triangular girder displays a much higher value of $C_{R L}$. Relative to the FGC, it is clear that the $C_{R L}$ of the bridge cases tremendously worsens. For the worst bridge case condition, there is an increase of $36.6 \%$ in the slender regime and a $21.1 \%$ increase in the bluff regime by comparison to the FGC.

\subsection{Flow Structure}

The visualization of flow structures surrounding the train area moving on different bridge configurations under various crosswind conditions are provided in this section in terms of streamlines of the time-averaged velocity field. The detailed flow structure of train moving on the FGC can be referred to Ishak et al., [10]. Detailed flow structures are depicted in two-dimensional illustrations from side views taken at $x / H=2$ from the train nose. As previously mentioned, the visualization of the flow structures are divided into two flow regimes (i.e. slender body flow regime and bluff body flow regime).

\subsubsection{Slender body flow regime}

\subsubsection{At $\Psi=0^{\circ}$ (No crosswind)}

Illustrations in Figure 12 are the free stream flows approaching the blunt nose train model that is positioned on top of different bridges. As can be seen, regardless of variation in shapes and configurations, at $\psi=0^{\circ}$, the flow structures surrounding the train are completely identical. The flow experiences separation along the leading edges, leading to the formation of different recirculation bubbles identical to the flat ground case (FGC). It is further confirmed by the values of global properties as shown in Figures 8 to 11 whereby all values attained for the vehicle moving on the bridges at $\Psi=0^{\circ}$ are exactly in parallel with the data obtained whilst the train is travelling on the FGC. $V_{1}, V_{4}$ and $V_{5}$ represent the vortices formed due to flow separation at the front leading edge of the train model and $S_{1}$ represents the stagnation area. 


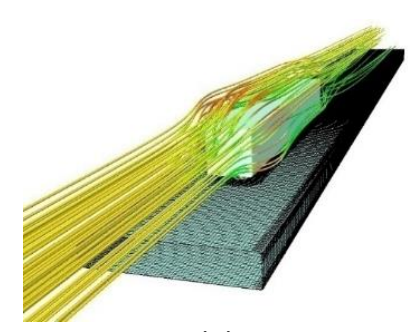

(a)

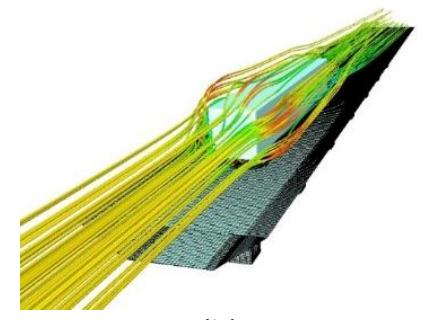

(b)

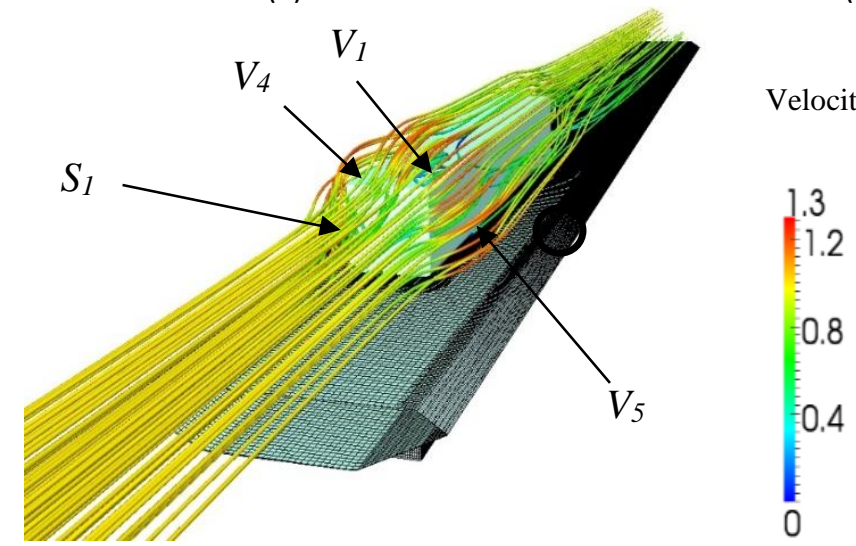

(c)

Fig. 12. Velocity streamlines passing the generic train at $\Psi=0^{\circ}$ for the bridge cases of (a) Rectangular girder (b) Triangular girder (c) Wedge girder

\subsubsection{At $\Psi=15^{\circ}$}

For the bridge with the rectangular girder, there are four pronounced vortices named $V_{(1 a)}, V_{(b)}$, $V_{(c)}$ and $V_{(d)}$ that influence the train aerodynamics as can be seen in Figure 13. As the flow passing the bridge, it is separated on the top leading edge of the girder and creates a recirculation region namely

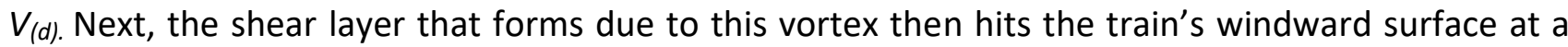
specific stagnation point named the half-saddle point denoted as S1/2. Starting from here, two parts of the flow are formed and oriented in two directions i.e. towards the roof surface and the bottom surface of the train model. Then, the upper part of the flow is separated once again starting at the

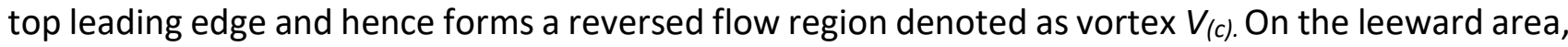
two recirculating flows (bubble) represented as $V_{(1 a)}$ and $V_{(b)}$ located above one another are detected. The flow structures on the bridge with the triangular girder resemble those of the rectangular girder. However, for the bridge with the wedge girder, due to its geometric difference, no flow separation is observed on the top of the deck (i.e. $V_{(d)}$ is absent). Thus, in this girder configuration case, the flow that hits the windward surface area is directly from the free stream flow. At this yaw angle condition, vortex core $V_{(c)}$ can be seen clearly, and it emphasizes the important characteristic of the slender body flow behaviour. In terms of aerodynamic loads, the much lower value of the drag force coefficient $\left(C_{d}\right)$ (see Figure 10) in the wedge girder case is justified due to the non-existence of vortex

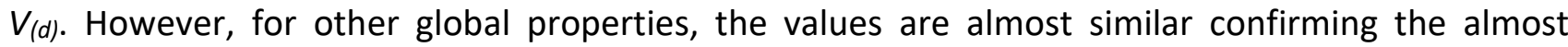
identical flow structures surrounding the train at $\psi=15^{\circ}$ for the different bridge girder conditions. 


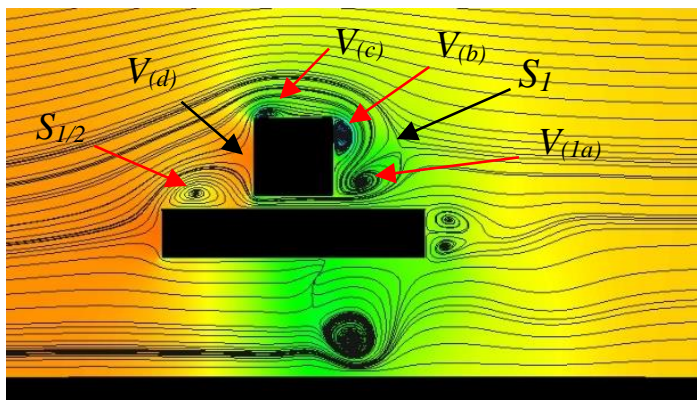

(a) Rectangular girder

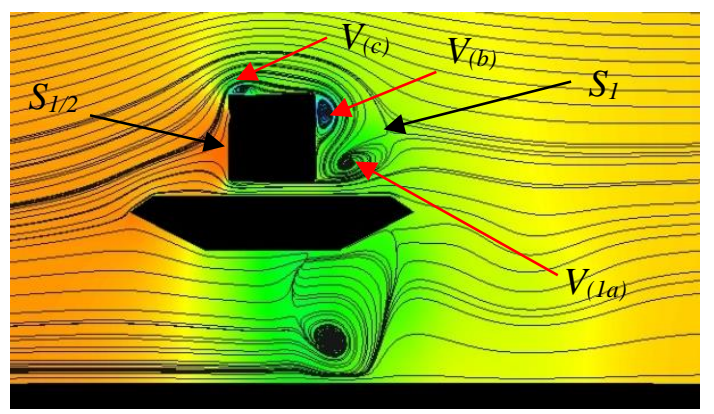

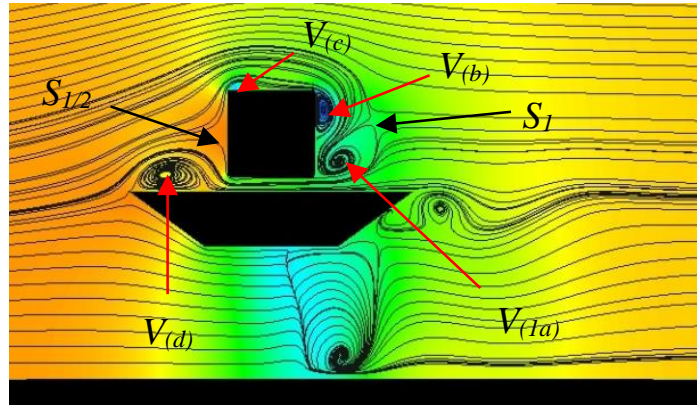

(b) Triangular girder

Pressure $\left(\frac{P}{\rho u_{\infty}{ }^{2}}\right)$

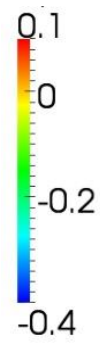

(c) Wedge girder

Fig. 13. Streamlines superimposed on the pressure contour for different bridge configurations at $x / H=2$ from the train's nose for $\psi=15^{\circ}$

\subsubsection{At $\Psi=30^{\circ}$}

At this crosswind condition, vortices especially on the leeward area start to intensify and become larger in size (see Figures 14(a)-(c)). For the rectangular girder, it is clear that vortex $V_{(b)}$ grows stronger than vortex $V_{(1 a)}$ when its existence almost leads to the suppression of vortex $V_{(1 a)}$. In the case of the wedge girder, vortex $V_{(1 a)}$ is already starting to shed away from its original position as shown in Figure 14(c). Other than that, vortex $V_{(c)}$ starts to enlarge and extends further towards the leeward top edge. Since there is still an existence of vortex core found for $V_{(c)}$, the flow behaviour is still considered as in the slender body flow regime. A much clearer view of vortices sliding on the leeward surface of the vehicle travelling on a bridge with rectangular girder shape is presented in Figure 15. To add on, the side force $\left(C_{s}\right)$ on the wedge girder case is the largest among other girder cases at this crosswind condition because of the increased area of higher-pressure region concentrated on the windward surface of the train. This is a consequence of no separation bubbles existed on the windward side of the vehicle like the other two configurations (i.e. rectangular girder and triangular girder). 


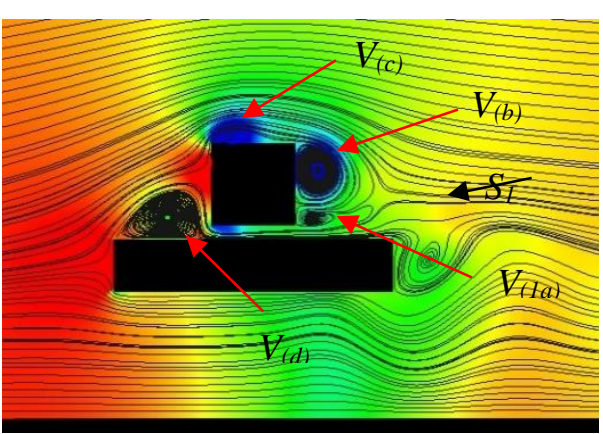

(a) Rectangular girder

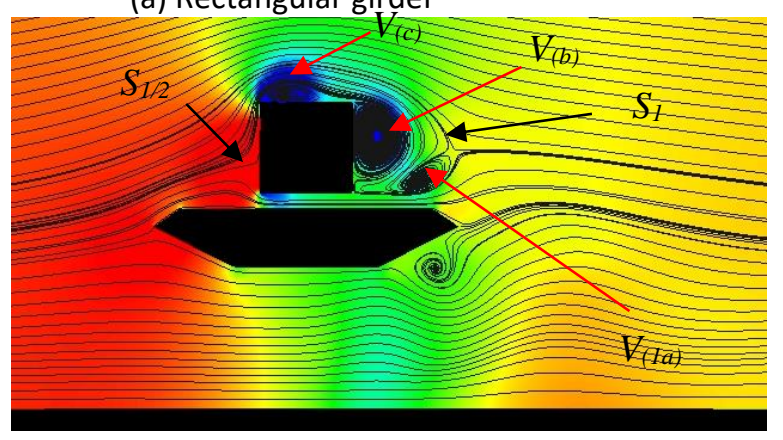

(c) Wedge girder

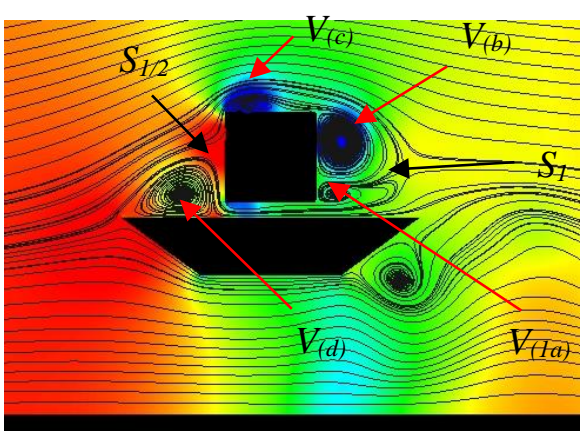

(b) Triangular girder

$$
\text { Pressure }\left(\frac{P}{\rho u_{\infty}{ }^{2}}\right)
$$

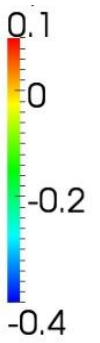

Fig. 14. Streamlines superimposed on the pressure contour for different bridge configurations at $x / H=2$ from the train's nose for $\psi=30^{\circ}$

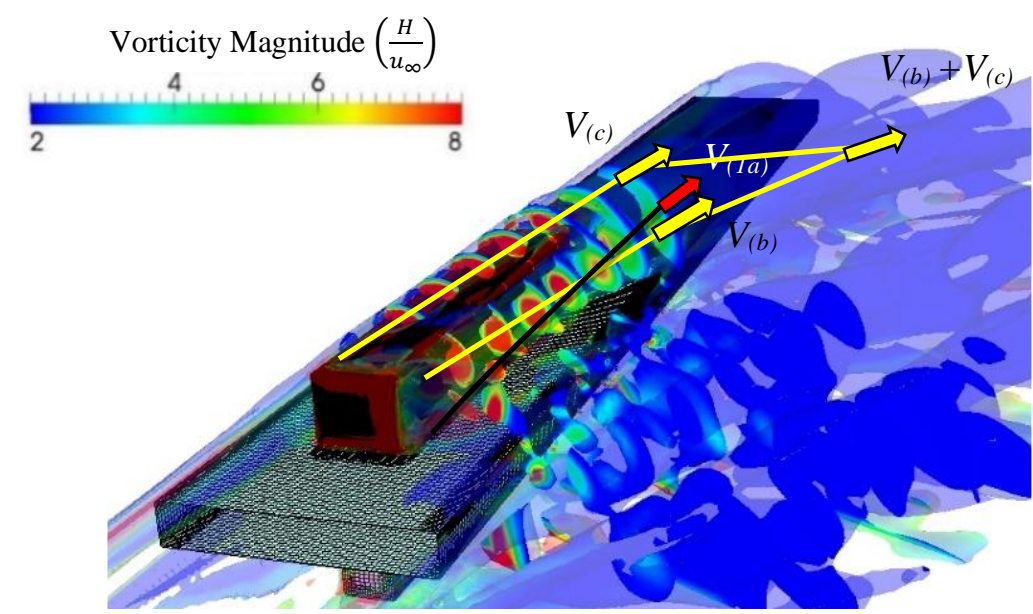

Fig. 15. Isosurfaces of $Q$-criterion for the train with the rectangular bridge girder at $\psi=30^{\circ}$

\subsubsection{Bluff body flow regime}

\subsubsection{At $\Psi=60^{\circ}$}

At $\Psi=60^{\circ}$, most of the bridge cases are already entering the bluff body flow regime. In this case, the characteristics of the bluff body flow behaviour follow with the big blunder shape of flow structures merging in the leeward area as shown clearly in Figure 16(a)-(c). Figure 17 illustrates the threedimensional flow structures for the bridge rectangular girder case where the merging of vortices happens in the leeward area. As can be seen from Figure 16(a), for the case of the rectangular girder, the core of vortex $V_{(c)}$ has disappeared after being rolled up with vortex $V_{(b)}$ in the leeward area. The merging of these vortices creates an expanded wake size that acquires a larger length (i.e. $2.62 \mathrm{H}$ ) elongated in the lateral direction. The same situation also applies for the wedge girder case as the 
core of vortex $V_{(c)}$ seemingly vanishes, eventually creating yet a new larger vortex (i.e. 2.42H). Interestingly, in the case of the triangular girder, vortex $V_{(c)}$ is still visible. This means that the flow is still in the transition phase from the slender body flow behaviour to the bluff body flow regime. This explains why the size of vortex formed in the leeward area is much smaller $(1.48 \mathrm{H})$ compared to the other two cases. As a relation with the aerodynamic loads, at $\psi=60^{\circ}$, the $C_{l}$ starts to become negative. This can be explained by the much lower pressure concentrated in the underbody area and thus inducing a downward force due to the direction in which the pressure is acting. The $C_{d}$ also decreases due to the low-pressure region that starts to drift away from the train body. Meanwhile, the $C_{s}$ starts to stabilize, and this confirms that the flow has entered the bluff body flow regime for most of the cases. The $C_{s}$ of the rectangular girder case is much lower because the vortices in the leeward area have less intensity. This is due to the position of the core vortex that is far away from the train's body on the leeward side.

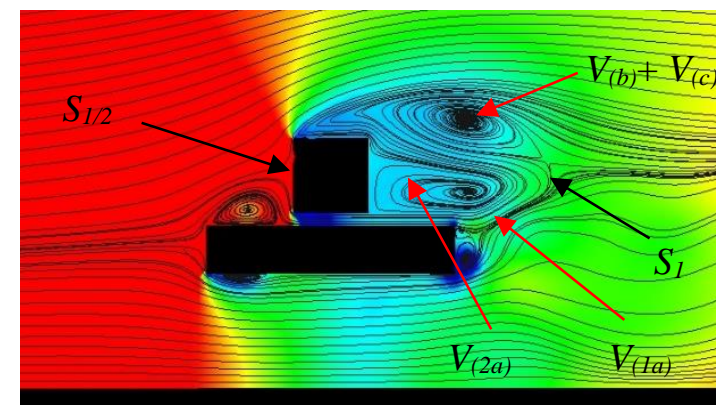

(a) Rectangular girder

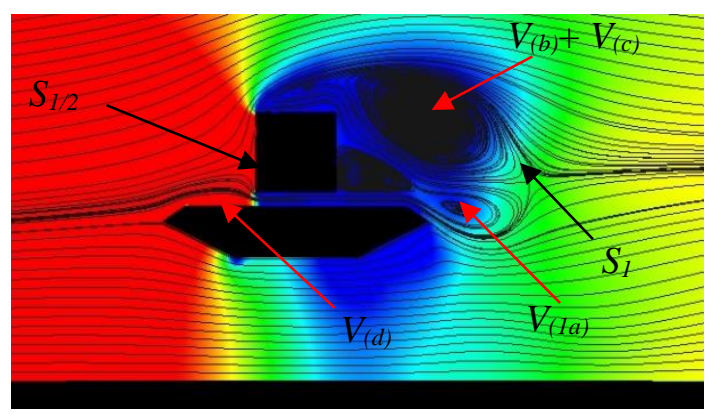

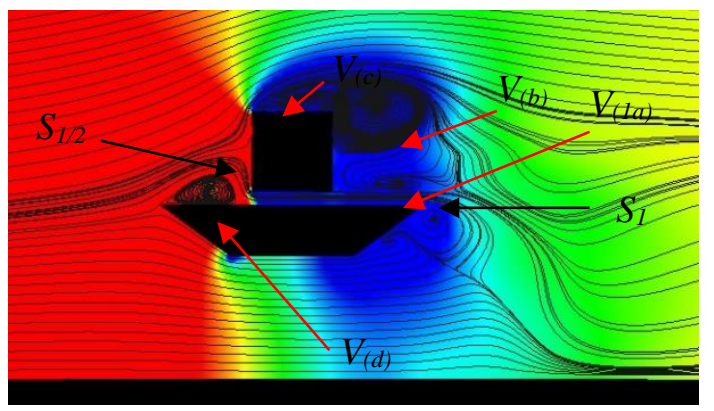

(b) Triangular girder

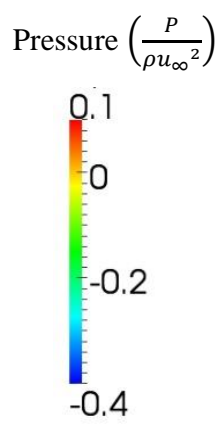

(c) Wedge girder

Fig. 16. Streamlines superimposed on the pressure contour for different bridge configurations at $x / H=2$ from the train's nose for $\psi=60^{\circ}$

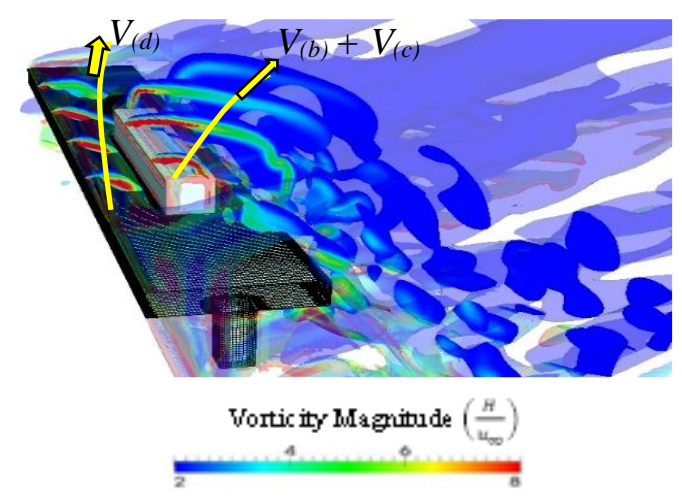

Fig. 17. Isosurfaces of $Q$-criterion at $\psi=$ $60^{\circ}$ for the train model on the top of the rectangular bridge girder 


\subsubsection{At $\Psi=75^{\circ}$}

All of the test cases can be said to experience the bluff body flow behaviour at $\psi=75^{\circ}$. Based on Figure 18, the wake structure on the leeward area has become larger i.e. $5.08 \mathrm{H}$ (rectangular girder), $3.25 \mathrm{H}$ (triangular girder), and $4.21 \mathrm{H}$ (wedge girder). The size of the vortex formed on the leeward side has significantly affected the pressure surrounding the area. The larger the vortex size, the further the vortex core moves from the train model. The leeward pressure region on the rectangular girder is higher compared to those on the wedge girder and the triangular girder. In terms of the $C_{s}$ outcomes (see Figure 8), the triangular girder experiences the highest side force loads, followed by the wedge girder and the rectangular girder. Additionally, at this condition, an interesting finding related to both wedge girder and triangular girder cases reveals that a secondary source vortex is seen coming from the bridge underbody (i.e. the blue pressure region underneath the leeward girder). As shown in Figures 18(b)-(c), this new vortex starts to suppress the development of the existing vortex at the train's le=;ard area. This is why the wake size is much smaller for both wedge girder and triangular girder cases. This happens due to the geometry of the girder shape that allows the wind flow to be directed upwards.

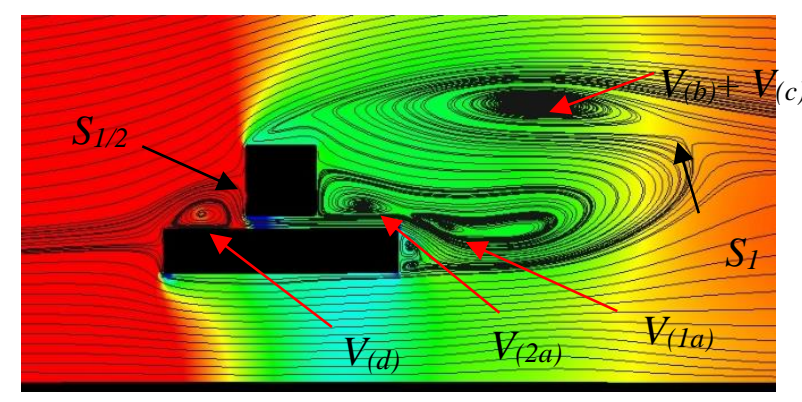

(a) Rectangular girder

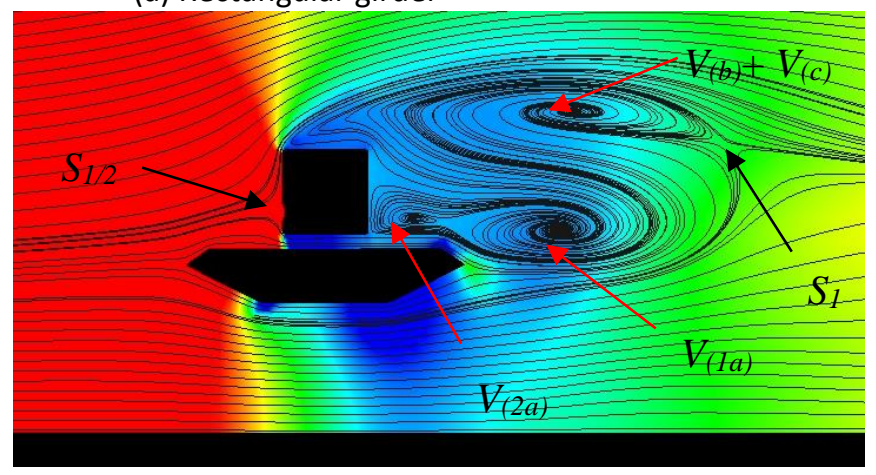

(c) Wedge girder

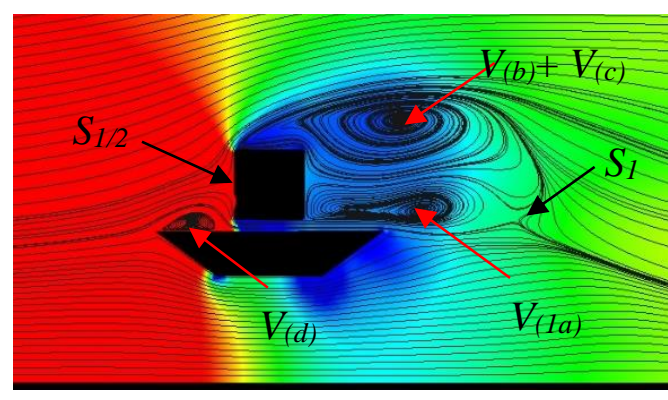

(b) Triangular girder

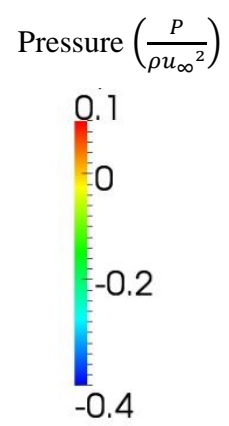

Fig. 18. Streamlines superimposed on the pressure contour for different bridge configurations at $x / H=2$ from the train's nose for $\Psi=75^{\circ}$

\subsubsection{At $\Psi=90^{\circ}$}

Figures 19(a)-(c) show a similar scenario where the leeward edge has entirely become the trailing edge. In the case of the rectangular girder, the vortex on the leeward side expands. However, the overall vortex size is significantly reduced for the triangular girder and wedge girder cases when compared to earlier crosswind scenarios. This is owing to the secondary source vortex $V_{s}$ observed emanating from the bridge underbody (see Figure 20(a)). This new vortex begins to inhibit the growth of the preexisting vortex at the train's leeward area. As a result, the wake size is significantly smaller in both wedge and triangular girder cases. This is due to the geometry of the girder design, which 
directs the wind flow upward and pushes the vortex $V_{s}$ in its path. Figures 19(b)-(c) depicted the flow on the leeward zone that is oriented uphill (note the arrows labelled as ' $A$ ') for a clearer picture. Vortex $V_{(d)}$ on the windward side, on the other hand, begins to suppress when the flow direction shifts from parallel to perpendicular for the rectangular and triangular girder cases, respectively. When examining the $C_{s}$ graph in Figure 8 , it is clear that the value increases slightly. As the leeward vortex core pushes closer to the vehicle surface, a considerably lower pressure zone forms on the train's leeward side, increasing $C_{s}$ values on both the triangular and wedge girders cases. Similarly, this situation has enabled for a rise in $C_{d}$.

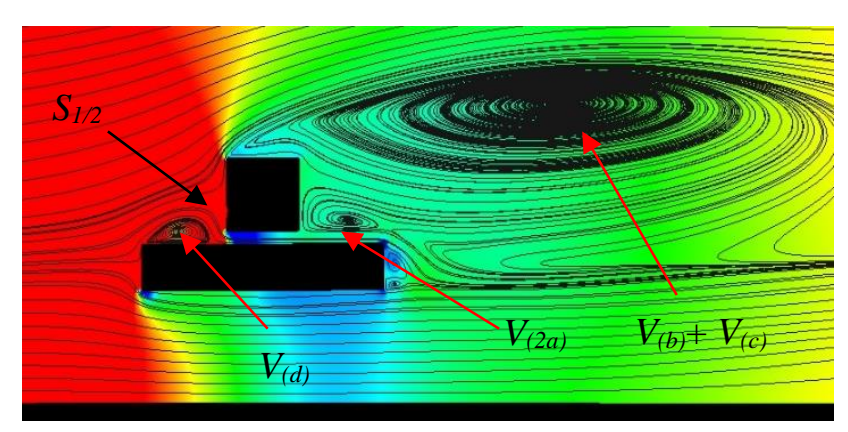

(a) Rectangular girder

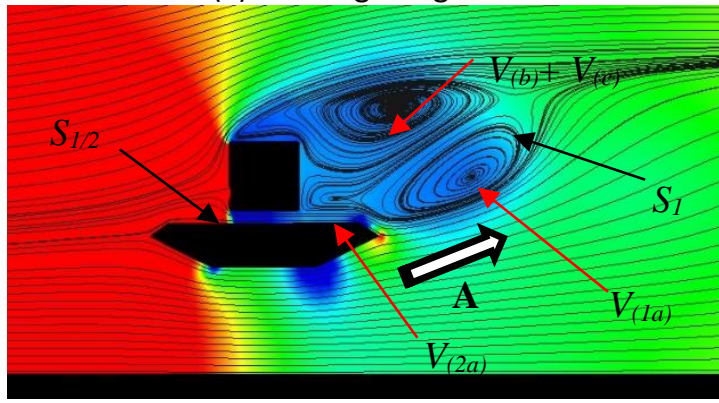

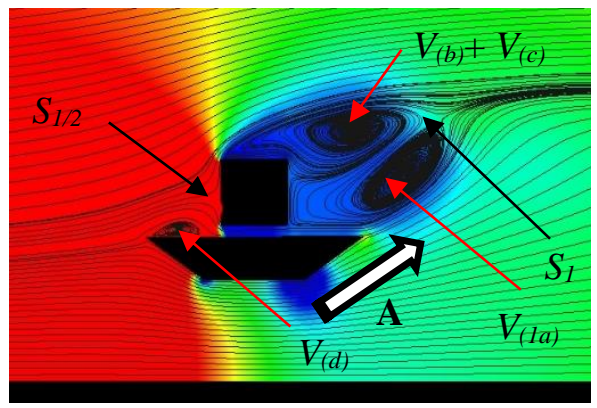

(b) Triangular girder

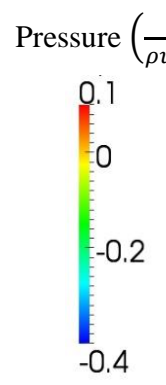

(c) Wedge girder

Fig. 19. Streamlines superimposed on the pressure contour for different bridge configurations at $x / H=2$ from the train's nose for $\Psi=90^{\circ}$

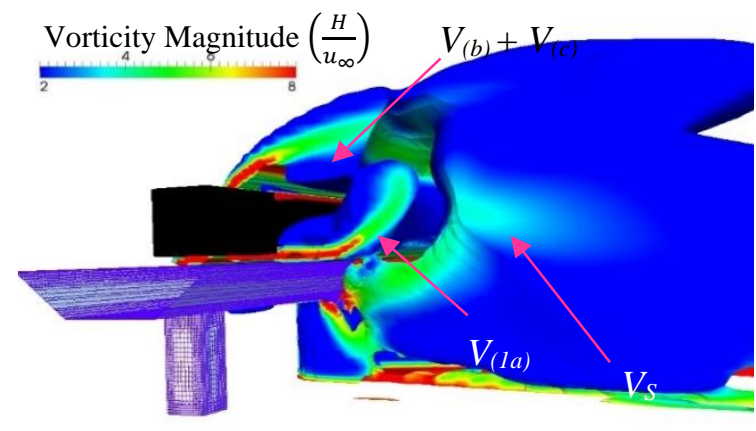

(a)

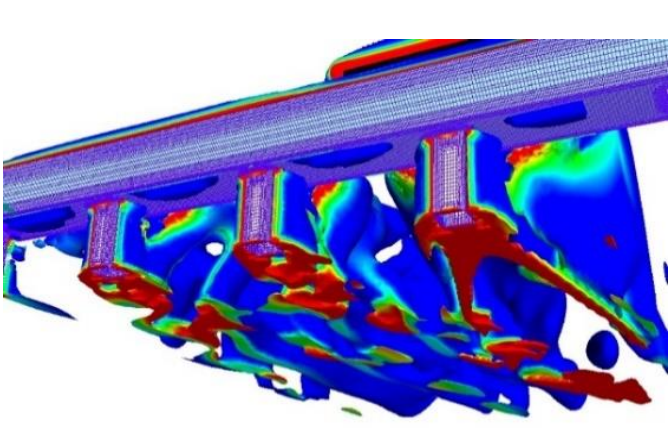

(b)

Fig.20. Isosurfaces of $Q$-criterion sliced through $x / H=2$ for the train vehicle on top of the triangular bridge girder at $\psi=90^{\circ}$ (a) from the front view (b) from the bottom view

\subsection{Pressure Distribution}

Results of the pressure coefficient $\left(C_{p}\right)$ at different positions along the cross-section taken at $x / H$ $=2$ from the train's nose are compared. The location of the cross-section is selected as it is where the 
flow is considered more developed as shown in flow structure discussions. Figure 21 shows the orientation of the train's circumference along which the $C_{p}$ was sampled.

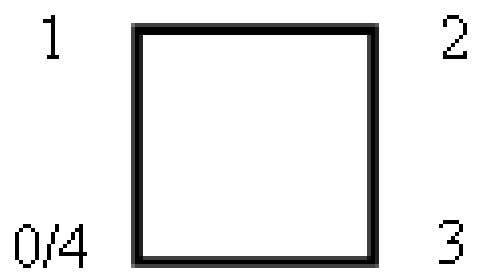

Fig. 21. Schematic of the train's circumference where four regions classified are: (a) Region I (0/4-1): Windward (b) Region II (1-2): Top (c) Region III (2-3): Leeward and (d) Region IV (3-0/4): Bottom

Generally, Figures 22(a)-(c) show that there are unique patterns representing the two flow regimes for the $C_{p}$ values for different bridge girder conditions. In the slender body flow regime ( $\psi \leq$ $\left.45^{\circ}\right)$, the $C_{p}$ values are inconsistent at Region II and Region III. On top of that, there is also another drop in the $C_{p}$ in Region IV due to separation at the bottom windward edge of the train model. In response to the higher yaw angle condition of $\psi \geq 60^{\circ}$ (i.e. bluff body flow regime), the pressures exerted on the train's body are more settled.

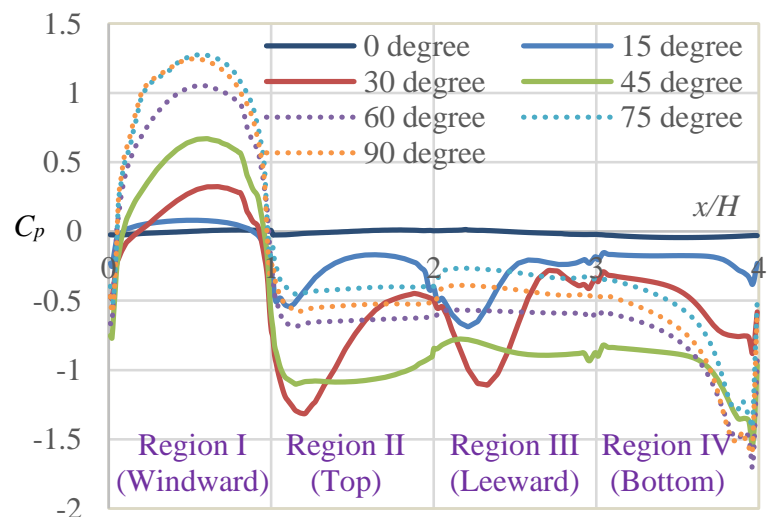

(a) Bridge: Rectangular girder

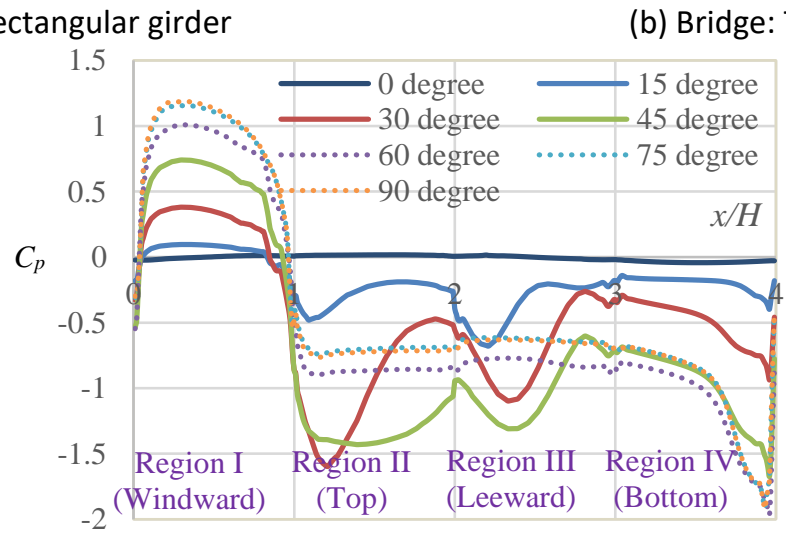

(c) Bridge: Wedge girder

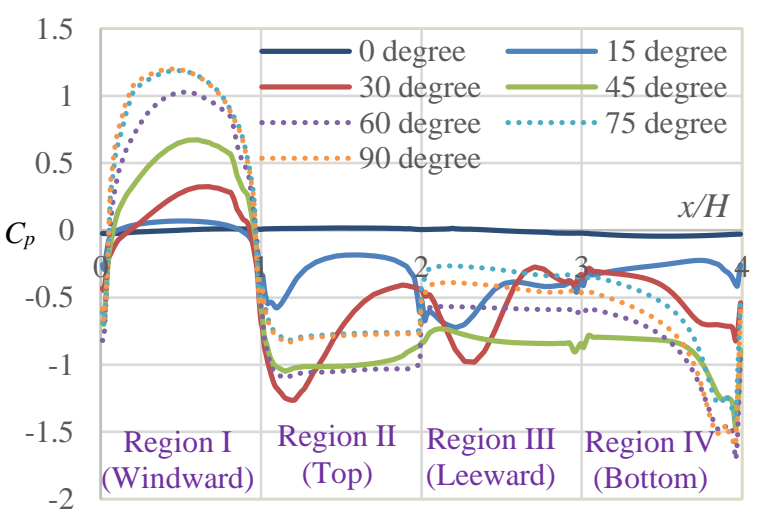

(b) Bridge: Triangular girder

Fig. 22. Pressure coefficient at $x / H=2$ from the train's nose for different bridge configurations 
When referring to the side force coefficient $\left(C_{s}\right)$ in Figure 8, the magnitude increases as the yaw angle increases. This can be described in Figures 22(a)-(c) by the differences in pressure coefficient $C_{p}$ for Region I (windward surface) and Region III (leeward surface). In the slender body flow regime, as the yaw angle increases, the pressure difference between Region I (windward surface) and Region III (leeward surface) becomes larger, as reflected by the results attained in Figure 8 . This is due to the high pressure exerted on the windward surface resulting in a much lower pressure region on the leeward area. However, as it enters the bluff body flow regime, the difference in the $C_{p}$ becomes less thus resulting in much more stable values of the $C_{s}$ at high crosswind conditions. On the other hand, from Figure 9 , the $C_{l}$ increases to a maximum value at $\psi=30^{\circ}$. This is justified from Figures Figure 22 whereby the largest difference in the $C_{p}$ occurs between Region II (top surface) and Region IV (bottom surface). Nonetheless, the downward force occurs in the bluff body flow regime as the direction of the lift force is in the opposite direction. This is because there is large pressure accumulated at the top surface of the train (Region II) compared to that on the bottom surface (Region IV).

\section{Conclusions}

This study has presented the investigations on the aerodynamic characteristics inclusive of associated global properties i.e. aerodynamic loads and the flow structures of a generic train model travelling on different bridges under the crosswind influence.

According to the findings, the aerodynamic loads are significantly impacted by both the crosswind yaw angle and the ground condition in which the train is travelling. Important parameters that have a significant impact on train stability, such as coefficient of side force $\left(C_{s}\right)$, rolling moment $\left(C_{R L}\right)$, lift force $\left(C_{l}\right)$, and drag force $\left(C_{d}\right)$, revealed that the bridge cases produced the worst results of these parameters when compared to the baseline scenario, i.e. the flat ground case (FGC). Loads for $C_{s}$ and $C_{R L}$ were critical at high yaw angles, with maximum values at $\psi=45^{\circ}$ and $=60^{\circ}$. In contrast, both $C_{l}$ and $C_{d}$ were deemed significantly more critical within a low range of yaw angles, with the highest value reported at $\psi=30^{\circ}$.

Furthermore, the evaluation of aerodynamic loads and flow structure can be categorised in terms of two distinct flow regime characteristics. The first flow regime is known as the slender body flow regime, and it occurs in the lower range of yaw angles (i.e. $\psi \leq 45^{\circ}$ ). The change in the $C_{S}$ and $C_{R L}$ was practically linear with the yaw angle in the slender regime. The graph for $C_{l}$ and $C_{d}$ showed a fluctuating rise to a maximum value before dropping. In terms of flow structures, the presence of individual vortex cores at each separation point is the fundamental feature of the flow indicating the slender regime flow behaviour. The second flow regime is known as the bluff body flow regime, and it occurs at larger yaw angles (i.e. $\psi \geq 60^{\circ}$ ). The aerodynamic loads (i.e. $C_{s}, C_{1}, C_{d}$, and $C_{R L}$ ) are less influenced by changes in crosswind yaw angles in this regime. Furthermore, the features of the bluff body flow regime are defined by the development of large separated flow regions caused by the merging of vortices on the train's leeward side. This resulted in the expansion of the vortices, which became more visible as the flow yaw angle increased. The physics of flow structures behaved similarly to the varied flow regime conditions in relation to the different bridge cases. However, when the flow structures are examined, the size dissimilarities of the vortices produced may be seen. This eventually corresponds to the previously noted differences in wake sizes in the leeward region. From the result obtained, the slender regime (i.e. $\psi \leq 45^{\circ}$ ) was predicted to have a wake size less than $x / H=2$, but the bluff regime (i.e. $\Psi \geq 60^{\circ}$ ) had a wake size more than $x / H=2$.

It was also observed that the pressure coefficient distribution $\left(C_{p}\right)$ around the circumference of the train surface at $x / H=2$ behaved differently according to the two flow regimes. More instability of the $C_{p}$ distribution occurred on the separation surface in the slender body flow regime whilst in 
the bluff body flow regime, the $C_{p}$ distribution was steadier in magnitude due to the merging of vortices. The observation was quite similar to the FGC. However, the magnitudes of the $C_{p}$ were slightly magnified. This happened especially at the windward surface of the train, and at the separation area with the bridge cases showed greater amplification compared to the FGC.

In a nutshell, the addition of infrastructure: bridge cases, along with the crosswind, will worsen the aerodynamic characteristics around the train as the geometries of the bridge girder were varied. The bridge with the wedge girder showed the worst aerodynamic properties with both important aerodynamic loads (i.e. side force and rolling moment), followed by the triangular girder and the rectangular girder. Hence, the study objectives have been successfully achieved. The study has effectively added to current knowledge in the field of train aerodynamics. In the future, the data obtained in the research could be useful in the determination of safety guidelines under which a train is moving on a bridge under different crosswind conditions. Train's daily operation system can then be further improved as the new safety guideline criteria emerged.

\section{Acknowledgement}

This research was financially supported by the Malaysia Ministry of Higher Education (MOHE) through Fundamental Research Grant Scheme (FRGS/1/2020/TK02/UTHM/03/4).

\section{References}

[1] Alam, Firoz, and Simon Watkins. "Crosswinds effects on high cube freight trains." In The third BSME-ASME thermal engineering conference, pp. 7-12. 2006.

[2] Zhang, Xlaoyu. Crosswind stability of vehicles under nonstationary wind excitation. Vol. 28. KIT Scientific Publishing, 2015.

[3] Diedrichs, Ben, Mikael Sima, A. Orellano, and Henrik Tengstrand. "Crosswind stability of a high-speed train on a high embankment." Proceedings of the Institution of Mechanical Engineers, Part F: Journal of Rail and Rapid Transit 221, no. 2 (2007): 205-225. https://doi.org/10.1243/0954409JRRT126

[4] Kamal, Muhammad Nabil Farhan, Izuan Amin Ishak, Nofrizalidris Darlis, Nor Afzanizam Samiran, Rahmah Mahmudin, Razlin Abd Rashid, Muhamad Asri Azizul, and Amir Khalid. "Effect of Crosswind on Aerodynamic Characteristics of a Generic Train Model using ANSYS." Journal of Industry, Engineering and Innovation 1, no. 1 (2019).

[5] S. M. Salleh, M. S. M. Ali, S. A. Z. Shaikh, I. A. Ishak, M. Shirakashi, and S. Muhammad. "Aerodynamics characteristics around simplified high speed train model under the effect of crosswinds." ARPN J. Eng. Appl. Sci. 12, no. 8 (2017).

[6] Nazri, Ahmad Nazirul, Izuan Amin Ishak, Nofrizalidris Darlis, Zuliazura Mohd Salleh, Shaiful Fadzil Zainal Abidin, and Syabillah Sulaiman. "Aerodynamic Analysis on the Effects of Different Type of Train's Nose Design using Ansys Software." Journal of Industry, Engineering and Innovation 1, no. 1 (2019).

[7] Sakri, F. Mohd, MS Mat Ali, and SAZ Sheikh Salim. "Computational investigations and grid refinement study of 3D transient flow in a cylindrical tank using OpenFOAM." In IOP Conference Series: Materials Science and Engineering, vol. 152, no. 1, p. 012058. IOP Publishing, 2016. https://doi.org/10.1088/1757-899X/152/1/012058

[8] Montenegro, P. A., R. Heleno, H. Carvalho, R. Calçada, and C. J. Baker. "A comparative study on the running safety of trains subjected to crosswinds simulated with different wind models." Journal of Wind Engineering and Industrial Aerodynamics 207 (2020): 104398. https://doi.org/10.1016/j.jweia.2020.104398

[9] Neto, José, Pedro Aires Montenegro, Cecília Vale, and Rui Calçada. "Evaluation of the train running safety under crosswinds-a numerical study on the influence of the wind speed and orientation considering the normative Chinese Hat Model." International Journal of Rail Transportation 9, no. 3 (2021): $204-231$. https://doi.org/10.1080/23248378.2020.1780965

[10] Ishak, Izuan Amin, Mohamed Sukri Mat Ali, Mohamad Fitri Mohd Yakub, and Sheikh Ahmad Zaki Shaikh Salim. "Effect of crosswinds on aerodynamic characteristics around a generic train model." International Journal of Rail Transportation 7, no. 1 (2019): 23-54. https://doi.org/10.1080/23248378.2018.1424573

[11] Ekeroth, F., N. Kalmteg, J. Pilqvist, and J. Runsten. "Crosswind flow around a high-speed train on embankment-an experimental and numerical study." PhD diss., Master's thesis, Chalmers University of Technology, The Institution of Applied Mechanics, Division of Fluid Mechanics, Gothenburg, Sweden (2009).

[12] Baker, C. J. "The simulation of unsteady aerodynamic cross wind forces on trains." Journal of wind engineering and industrial aerodynamics 98, no. 2 (2010): 88-99. https://doi.org/10.1016/j.jweia.2009.09.006 
[13] Proppe, Carsten, and Christian Wetzel. "A probabilistic approach for assessing the crosswind stability of ground vehicles." Vehicle system dynamics 48, no. S1 (2010): 411-428. https://doi.org/10.1080/00423114.2010.482158

[14] Yu, Mengge, Jiali Liu, Dawei Liu, Huanming Chen, and Jiye Zhang. "Investigation of aerodynamic effects on the highspeed train exposed to longitudinal and lateral wind velocities." Journal of Fluids and Structures 61 (2016): $347-$ 361. https://doi.org/10.1016/j.jfluidstructs.2015.12.005

[15] Suzuki, Minoru, Katsuji Tanemoto, and Tatsuo Maeda. "Aerodynamic characteristics of train/vehicles under cross winds." Journal of wind engineering and industrial aerodynamics 91, no. 1-2 (2003): $209-218$. https://doi.org/10.1016/S0167-6105(02)00346-X

[16] Sanquer, Stephane, Christian Barre, Marc Dufresne de Virel, and Louis-Marie Cleon. "Effect of cross winds on highspeed trains: development of a new experimental methodology." Journal of Wind Engineering and Industrial Aerodynamics 92, no. 7-8 (2004): 535-545. https://doi.org/10.1016/i.jweia.2004.03.004

[17] Thomas, Dirk. "Lateral stability of high-speed trains at unsteady crosswind." PhD diss., KTH Farkost \& Flyg, 2009.

[18] Ishak, Izuan Amin, Mohamed Sukri Mat Ali, Fadhilah Mohd Sakri, Fathul Hakim Zulkifli, Nofrizalidris Darlis, Rahmah Mahmudin, Shaiful Fadzil Zainal Abidin et al. "Aerodynamic Characteristics Around a Generic Train Moving on Different Embankments under the Influence of Crosswind." Journal of Advanced Research in Fluid Mechanics and Thermal Sciences 61, no. 1 (2019): 106-128.

[19] I. A. Ishak, R. Mahmudin, N. Samiran, Z. Salleh, and N. Darlis. "Safety guideline for a generic train travelling on different platform scenarios under the influence of crosswind." Int. J. Adv. Trends Comput. Sci. Eng. 9, no. 1.4 (2020): 138-150. https://doi.org/10.30534/ijatcse/2020/2191.42020

[20] Olmos, J. M., and M. A. Astiz. "Improvement of the lateral dynamic response of a high pier viaduct under turbulent wind during the high-speed train travel." Engineering Structures 165 (2018): 368-385. https://doi.org/10.1016/i.engstruct.2018.03.054

[21] Andersson, Evert, J. Häggström, Mikael Sima, and Sebastian Stichel. "Assessment of train-overturning risk due to strong cross-winds." Proceedings of the Institution of Mechanical Engineers, Part F: Journal of rail and rapid transit 218, no. 3 (2004): 213-223. https://doi.org/10.1243/0954409042389382

[22] Baker, C. J. "The determination of topographical exposure factors for railway embankments." Journal of Wind Engineering and Industrial Aerodynamics 21, no. 1 (1985): 89-99. https://doi.org/10.1016/0167-6105(85)90035-2

[23] Montenegro, P. A., D. Barbosa, H. Carvalho, and R. Calçada. "Dynamic effects on a train-bridge system caused by stochastically generated turbulent wind fields." Engineering Structures 211 (2020): 110430. https://doi.org/10.1016/i.engstruct.2020.110430

[24] Cheli, Federico, Roberto Corradi, Daniele Rocchi, Gisella Tomasini, and Emilio Maestrini. "Wind tunnel tests on train scale models to investigate the effect of infrastructure scenario." Journal of Wind Engineering and Industrial Aerodynamics 98, no. 6-7 (2010): 353-362. https://doi.org/10.1016/j.jweia.2010.01.001

[25] Bocciolone, Marco, Federico Cheli, Roberto Corradi, Sara Muggiasca, and Gisella Tomasini. "Crosswind action on rail vehicles: Wind tunnel experimental analyses." Journal of wind engineering and industrial aerodynamics 96, no. 5 (2008): 584-610. https://doi.org/10.1016/j.jweia.2008.02.030

[26] Zhou, Dan, Hong-qi Tian, Mark Thompson, and John Sheridan. "Numerical and Experimental Investigations of the Flow Around a High-Speed Train on an Embankment Under Side Wind Conditions." In International Conference on Engineering Conferences International, pp. 113-130. Springer, Cham, 2010. https://doi.org/10.1007/978-3-31920122-1 7

[27] Xiang, Chao Qun, Wen Hua Guo, and Jia Wen Zhang. "Study on numerical simulation method of aerodynamic performance of high-speed train on bridge." In Applied mechanics and materials, vol. 376, pp. 312-316. Trans Tech Publications Ltd, 2013. https://doi.org/10.4028/www.scientific.net/AMM.376.312

[28] Xiang, Chao Qun, Wen Hua Guo, and Jia Wen Zhang. "Study on critical speed of high-speed train running on bridge under strong crosswind." In Applied Mechanics and Materials, vol. 501, pp. 1157-1161. Trans Tech Publications Ltd, 2014. https://doi.org/10.4028/www.scientific.net/AMM.501-504.1157

[29] Östh, Jan, and Sinisa Krajnovic. "Simulations of flow around a simplified train model with a drag reducing device." In Proceedings from Conference on Modelling fluid flow CMFF'12 (edited by J. Vad), September 4-7, 2012, Budapest. 2012.

[30] Hemida, Hassan, and Siniša Krajnović. "Exploring flow structures around a simplified ICE2 train subjected to a 30 side wind using LES." Engineering applications of computational fluid mechanics 3, no. 1 (2009): 28-41. https://doi.org/10.1080/19942060.2009.11015252

[31] Liu, Yebo, Hassan Hemida, and Zhiming Liu. "Large eddy simulation of the flow around a train passing a stationary freight wagon." Proceedings of the Institution of Mechanical Engineers, Part F: Journal of Rail and Rapid Transit 228, no. 5 (2014): 535-545. https://doi.org/10.1177/0954409713488096

[32] Sakuma, Yutaka, and Atsushi Ido. "Wind tunnel experiments on reducing separated flow region around front ends of vehicles on meter-gauge railway lines." Quarterly Report of RTRI50, no. 1 (2009): 20-25. 
https://doi.org/10.2219/rtriqr.50.20

[33] Wang, Qi, Haili Liao, Mingshui Li, and Cunming Ma. "Influence of aerodynamic configuration of a streamline box girder on bridge flutter and vortex-induced vibration." Journal of Modern Transportation 19, no. 4 (2011): 261-267. https://doi.org/10.1007/BF03325767

[34] Bruno, Luca, and Giuseppe Mancini. "Importance of deck details in bridge aerodynamics." Structural Engineering International 12, no. 4 (2002): 289-294. https://doi.org/10.2749/101686602777965234

[35] Biadgo, Asress Mulugeta, Aleksandar Simonović, Jelena Svorcan, and Slobodan Stupar. "Aerodynamic characteristics of high speed train under turbulent cross winds: A numerical investigation using unsteady-RANS method." FME Transactions 42, no. 1 (2014): 10-18. https://doi.org/10.5937/fmet1401010B

[36] Hemida, Hassan, and Siniša Krajnović. "LES study of the influence of the nose shape and yaw angles on flow structures around trains." Journal of Wind Engineering and Industrial Aerodynamics 98, no. 1 (2010): 34-46. https://doi.org/10.1016/i.jweia.2009.08.012

[37] Celik, Ishmail B., Urmila Ghia, Patrick J. Roache, and Christopher J. Freitas. "Procedure for estimation and reporting of uncertainty due to discretization in CFD applications." Journal of fluids Engineering-Transactions of the ASME 130, no. 7 (2008). https://doi.org/10.1115/1.2960953

[38] Ali, Mohamed Sukri Mat, Con J. Doolan, and Vincent Wheatley. "Grid convergence study for a two-dimensional simulation of flow around a square cylinder at a low Reynolds number." In Seventh International Conference on CFD in The Minerals and Process Industries (ed. PJ Witt \& MP Schwarz), pp. 1-6. 2009.

[39] Maruai, Nurshafinaz Mohd, Mohamed Sukri Mat Ali, Mohamad Hafiz Ismail, and Sheikh Ahmad Zaki. "Flow-induced vibration of a square cylinder and downstream flat plate associated with micro-scale energy harvester." Journal of Wind Engineering and Industrial Aerodynamics 175 (2018): 264-282. https://doi.org/10.1016/i.jweia.2018.01.010

[40] Ishak, Izuan Amin, Mohamed Sukri Mat Ali, and Sheikh Ahmad Zaki Shaikh Salim. "Mesh size refining for a simulation of flow around a generic train model." Wind and Structures 24, no. 3 (2017): 223-247. https://doi.org/10.12989/was.2017.24.3.223

[41] Samion, Siti Ruhliah Lizarose, Mohamed Sukri Mat Ali, Aminudin Abu, Con J. Doolan, and Ric Zong-Yang Porteous. "Aerodynamic sound from a square cylinder with a downstream wedge." Aerospace Science and Technology 53 (2016): 85-94. https://doi.org/10.1016/i.ast.2016.03.007

[42] Baker, C. J. "Ground vehicles in high cross winds part I: Steady aerodynamic forces." Journal of Fluids and Structures 5, no. 1 (1991): 69-90. https://doi.org/10.1016/0889-9746(91)80012-3 